\title{
'Ideally' unstable current sheets and the triggering of fast magnetic reconnection
}

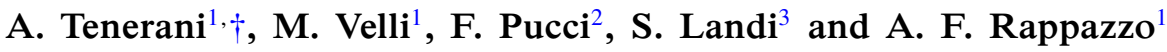 \\ ${ }^{1}$ EPSS, University of California, Los Angeles, CA 90095, USA \\ ${ }^{2}$ Dipartimento di Fisica, Università di Roma-Tor Vergata, I-00133 Rome, Italy \\ ${ }^{3}$ Dipartimento di Fisica e Astronomia, Università degli Studi di Firenze, I-50125 Florence, Italy
}

(Received 12 December 2015; revised 20 September 2016; accepted 21 September 2016)

Magnetic reconnection is thought to be the dynamical mechanism underlying many explosive phenomena observed both in space and in the laboratory, although the question of how fast magnetic reconnection is triggered in such high Lundquist $(S)$ number plasmas has remained elusive. It has been well established that reconnection can develop over time scales faster than those predicted traditionally once kinetic scales are reached. It has also been shown that, within the framework of resistive magnetohydrodynamics (MHD), fast reconnection is achieved for thin enough sheets via the onset of the so-called plasmoid instability. The latter was discovered in studies specifically devoted to the Sweet-Parker current sheet, either as an initial condition or an apparent transient state developing in nonlinear studies. On the other hand, a fast tearing instability can grow on an ideal, i.e. $S$-independent, time scale (dubbed 'ideal' tearing) within current sheets whose aspect ratio scales with the macroscopic Lundquist number as $L / a \sim S^{1 / 3}$ - much smaller than the Sweet-Parker one - suggesting a new way to approach to the initiation of fast reconnection in collapsing current configurations. Here we present an overview of what we have called 'ideal' tearing in resistive MHD, and discuss how the same reasoning can be extended to other plasma models commonly used that include electron inertia and kinetic effects. We then discuss a scenario for the onset of 'ideal' fast reconnection via collapsing current sheets and describe a quantitative model for the interpretation of the nonlinear evolution of 'ideally' unstable sheets in two dimensions.

Key words: plasma instabilities, plasma nonlinear phenomena, space plasma physics

\section{Introduction}

Magnetic reconnection is a process whereby magnetic energy is converted locally into particle heat and kinetic energy via some mechanism of effective magnetic dissipation that allows for a change of magnetic field line connectivity. Magnetic reconnection is ubiquitous in space and laboratory plasmas, and is believed to be at the heart of many observed phenomena, such as solar flares (Masuda et al. 1994; Su et al. 2013), geomagnetic substorms (Angelopoulos et al. 2013) and sawtooth crashes in tokamaks (Kadomtsev 1975; Yamada et al. 1994). Apart from these transient events,

$\dagger$ Email address for correspondence: annatenerani@epss.ucla.edu 
reconnection is also invoked in coronal heating models in different extensions of the nanoflare scenario (Parker 1988; Rappazzo et al. 2008), and plays a fundamental role during dynamo processes in primordial galaxy clusters (Schekochihin et al. 2005).

Several phenomena in which magnetic reconnection is thought to take place exhibit an explosive character, in the sense that magnetic energy can be stored over a long period of time and then suddenly released on a time scale comparable with the macroscopic ideal Alfvén time $\tau_{A}=L / v_{A}$, where $L$ is the macroscopic length of the system and $v_{A}=B_{0} / \sqrt{4 \pi \rho_{0}}$ the Alfvén speed defined through typical values of magnetic field magnitude $B_{0}$ and plasma mass density $\rho_{0}$. For many years, studies of reconnection stumbled on understanding how fast reconnection is triggered. The major difficulty came from the fact that the traditional models of reconnection stemming from the original Sweet-Parker mechanism (Parker 1957; Sweet 1958) or from the instability of macroscopic current sheets (Furth, Killeen \& Rosenbluth 1963), dating back to the 1960s, were clearly inadequate to explain the observed sudden release of magnetic energy, as such models predict magnetic reconnection time scales scaling with a positive power of $S$, where $S=L v_{A} / \eta \simeq 10^{6}-10^{14}$ is the macroscopic Lundquist number, $\eta$ being the magnetic diffusivity - that are far too long to be of any consequence. Several attempts involved locally enhancing the value of diffusivity by invoking anomalous resistivities to make the Sweet-Parker current layer transition to the fast, steady-state Petschek configuration (Petschek 1964). However, as discussed, e.g. in Shibata \& Tanuma (2001), these also require the formation of extremely small scales in the plasma.

The aim of the present review is to discuss how the difficulty of apparently slow reconnection has been overcome, following the works of Biskamp (1986), studies of the plasmoid instability (Loureiro, Schekochihin \& Uzdensky 2007) and the fractal reconnection scenario introduced by Shibata \& Tanuma (2001), with emphasis on research carried out by the present authors and in particular on the 'ideal' tearing scenario introduced in Pucci \& Velli (2014). Magnetic reconnection has been the subject of intense research both theoretically and observationally, and in very different physical as well as astrophysical contexts. A complete review on the subject would go well beyond the purpose of the present paper. Here we have tried to include most of the recent papers pertaining to fast reconnection, although the discussion may be brief. A longer review can however be found for instance in Yamada, Kulsrud \& Ji (2010).

Over the past decades a vast body of literature has focussed on what might accelerate reconnection speed up to realistic values. For the most part, these works approach the problem by studying (two-dimensional) magnetic reconnection at a single $\mathrm{X}$-point, usually imposed by deforming an initially thick current sheet. The ensuing dynamics at the X-point (sometimes called the developmental phase) eventually leads to an inner current sheet (or diffusion region), in which reconnection is studied by assuming a steady state (or asymptotic phase) is reached. Two major scenarios for onset of fast reconnection have emerged in this way (see also Daughton \& Roytershteyn 2012; Cassak \& Drake 2013; Huang \& Bhattacharjee 2013; Loureiro \& Uzdensky 2015).

In resistive magnetohydrodynamics (MHD), numerical simulations show that slowly reconnecting current sheets reminiscent of the Sweet-Parker model (SP) arise from the X-point. The basic SP configuration is shown in figure 1: the current sheet has an inverse aspect ratio $a_{S P} / L \sim S^{-1 / 2}$, maintained by a continuous inflow of plasma at speed $u_{i n} \ll v_{A}$ which convects the upstream magnetic field $\boldsymbol{B}_{0}$ into the diffusion region, and by an outflow at speed $u_{\text {out }} \simeq v_{A}$ which drags the reconnected magnetic 


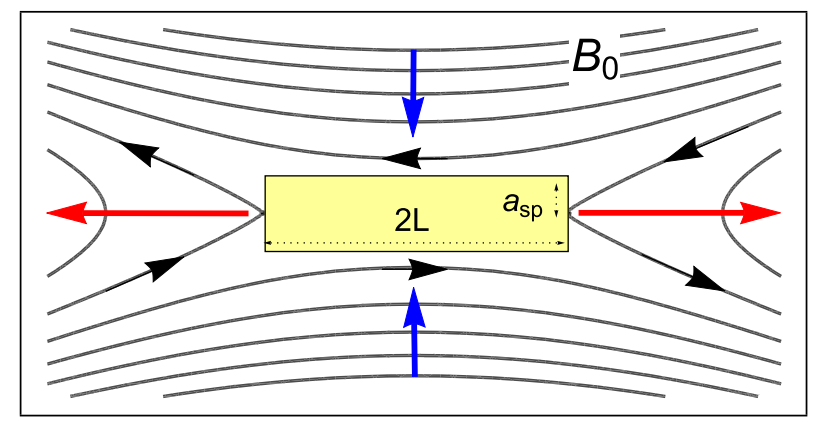

FIGURE 1. Sweet-Parker model. The diffusion region, in yellow, has an inverse aspect ratio $a_{S P} / L$. Coloured arrows represent the plasma flow into and outward the diffusion region.

field lines outwards, along the sheet; the resulting Alfvén-normalized reconnection rate is $u_{\text {in }} / u_{\text {out }} \sim a_{S P} / L \sim S^{-1 / 2}$, assuming the steady-state configuration remains stable. Once computational power allowed to study systems at larger values of $S$ $\left(S \gtrsim 10^{4}\right)$, it became clear that SP-like sheets become unstable to tearing (Biskamp 1986, 2000), the latter inducing the growth of a large number of magnetic islands (plasmoids). In this regard, linear stability analysis shows that SP sheets are highly unstable to a super-Alfvénic tearing instability - with a growth rate scaling with a positive power of $S, \gamma_{S P} \tau_{A} \sim S^{1 / 4}$ - as convincingly proved by Loureiro et al. (2007), Loureiro, Schekochihin \& Uzdensky (2013). Though such an instability demonstrates the possibility of fast reconnection already in resistive MHD, Pucci \& Velli (2014) pointed out that the growth rate, which diverges in the ideal limit $S \rightarrow \infty$, poses consistency problems, since magnetic reconnection is prohibited in ideal MHD. In other words, they questioned the realizability of current sheets with excessively large aspect ratios, such as SP, which, when taking the ideal limit starting from resistive MHD, reach an infinite growth rate, i.e. become unstable on time scales which at large enough $S$ are much shorter than any conceivable dynamical time required to set-up the corresponding configuration. Although this issue of reconnection speed was implicitly recognized in Loureiro et al. (2007) and many other works, (Bhattacharjee et al. 2009; Cassak \& Drake 2009; Samtaney et al. 2009; Huang \& Bhattacharjee 2010; Uzdensky, Loureiro \& Schekochihin 2010; Loureiro et al. 2012; Ni et al. 2015) the discussion of the onset of fast tearing has mostly remained anchored to the Sweet-Parker sheet framework.

Alternatively, it has been suggested that if ion scales are of the order of, or larger than, the thickness of the SP sheet, then two-fluid effects enhance the reconnection rate via what is usually called Hall-mediated reconnection. It was already known that the Hall term in Ohm's law increases the reconnection speed because of the excitation of dispersive waves, e.g. whistler waves (Terasawa 1983; Mandt, Denton \& Drake 1994; Biskamp, Schwartz \& Drake 1995; Rogers et al. 2001). To be more specific, it was argued, on the basis of numerical simulations, that large reconnection rates should be achieved during the nonlinear asymptotic phase, regardless of the mechanisms allowing reconnection (e.g. resistivity or electron inertia in collisionless reconnection), essentially because the Hall term modifies the structure of the diffusion region that becomes localized at scales of the order of the ion inertial length (Shay et al. 1999, 2004; Cassak, Shay \& Drake 2005; Drake, Shay \& Swisdak 
2008; Shepherd \& Cassak 2010). However, there is still no general agreement on whether and how the reconnection rate depends on the system size $L$ and plasma parameters such as the ion and electron inertial length $d_{i}$ and $d_{e}$ or resistivity, in the presence of the Hall term (Porcelli et al. 2002; Battacharjee, Germaschewski \& Ng 2005). More recent numerical results from PIC (particle in cell) simulations have also cast doubt on the necessity of exciting dispersive waves to reach higher reconnection rates (Liu et al. 2014). The robustness of the steady-state configuration reached during the asymptotic phase, and seen in several numerical studies (Birn et al. 2001; Shepherd \& Cassak 2010), has been called into question by both PIC and Hall-MHD simulations employing open boundaries or larger system sizes (Daughton, Scudder \& Karimabadi 2006; Klimas, Hesse \& Zenitani 2008; Huang, Bhattacharjee \& Sullivan 2011). These works provide some evidence that a final steady-state configuration may not always exist in Hall reconnection, and that the thin sheet constituting the diffusion region tends to stretch along the outflow direction until it becomes unstable to generation of secondary plasmoids. This would point to a strong analogy with the dynamics of thin current sheets found in resistive MHD.

Linear analysis of the tearing mode in resistive MHD proves that there exists a critical current sheet with an inverse aspect ratio $a_{i} / L \sim S^{-1 / 3}$ (Pucci \& Velli 2014), hence much larger than the SP one, that separates slowly reconnecting sheets (growth rate scaling with a negative power of $S$ ), from those exhibiting super-Alfvénic plasmoid instabilities (growth rate diverging with $S$ ), and that this has the proper convergence properties to ideal MHD: critical current sheets are unstable to a tearing mode growing at a rate independent from $S$ and, in this sense, the instability is 'ideal'. The existence of 'ideal' tearing therefore implies the impossibility of constructing any configuration corresponding to sheets thinner than critical, such as the paradigmatic Sweet-Parker sheet, suggesting a different route to the triggering of fast reconnection. At the same time, as we shall see, reasoning in terms of the rescaling arguments provides a clear predictive pathway to the critical events involved in a dynamics that will trigger fast reconnection, providing an alternative framework within which the many and different results previously obtained in simulations may be reinterpreted. It is worth mentioning that one of the major difficulties in this kind of numerical simulation concerns achieving sufficiently large Lundquist numbers. At the intermediate Lundquist numbers usual to MHD simulations, say $S \simeq 10^{4}-10^{6}$, the SP sheet is at most 10 times thinner than the critical current sheet predicted by the 'ideal' tearing theory. Moreover, the possible presence of plasma flows along the current sheet tends to stabilize the tearing mode (Bulanov, Syrovatskir \& Sakai 1978), inducing the formation of more elongated current sheets, that is, of layers having inverse aspect ratios smaller than $S^{-1 / 3}$ (in the resistive case). Therefore, with the Lundquist number not sufficiently large, the distinction between 'ideal' tearing framework and SP-plasmoids might be hard to observe, although the departure from the SP-plasmoid framework should become increasingly obvious with increasing $S$.

Throughout this review we summarize and complement recent results stemming from the 'ideal' tearing idea, obtained from both linear theory and nonlinear numerical simulations, providing a coherent perspective on recent studies and their relation to previous models: 'ideal' tearing can explain the trigger of fast reconnection occurring on critically unstable current sheets and can provide a guide - at least in two dimensions - to the nonlinear evolution with a model that describes the different stages of its evolution. Although we focus mainly on the resistive MHD description of the plasma, the 'ideal' tearing idea can be extended to other regimes, such as two fluid, Hall-MHD and so on to completely kinetic ones, providing a unified and 


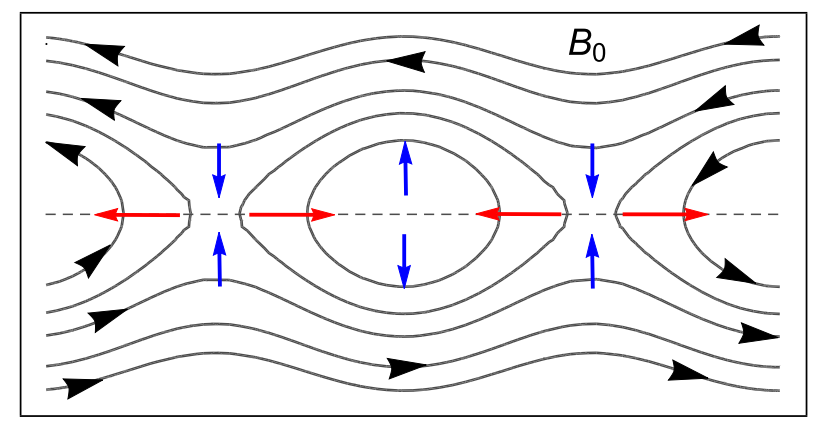

FIGURE 2. Tearing mode instability. Coloured arrows represent the perturbed plasma flows into and outward the X-point.

self-consistent framework for the onset of fast reconnection. In this paper we briefly discuss the application to simple kinetic models of collisionless reconnection.

The paper is organized as follows: in $\S 2$ we summarize the theory of the tearing mode instability in its traditional form, in order to prepare the ground for the 'ideal' tearing, that we discuss in $\S 3$; in $\S 4$ we approach the problem of the trigger of fast reconnection and we propose a new scenario relying on the 'ideal' tearing; in $\S 5$ we show that the trigger of fast reconnection via 'ideal' tearing can describe the evolution of a disrupting current sheet during its different nonlinear stages, characterized by a recursive sequence of tearing instabilities, and we compare our results with previous existent models in $\S 6$; a final summary and discussion are deferred to $\S 7$. For the sake of clarity, we mention here that in $\$ \S 2$ and 3 we show results that have been obtained from linear theory by solving numerically the system of ordinary differential equations of the eigenvalue problem of the tearing mode, with an adaptive finite difference scheme based on Newton iteration (Lentini \& Pereira 1987); in $\$ \S 4$ and 5 we show results obtained from 2 and 1/2 dimensional, fully nonlinear resistive MHD simulations.

\section{Background: the tearing mode instability}

Magnetic reconnection can arise spontaneously within current sheets as the outcome of an internal tearing instability when the non-ideal terms in Ohm's law, resistivity in our case, are taken into account. Tearing modes have been extensively studied in the simpler case of an infinite (one-dimensional) sheet both in the linear (Furth et al. 1963) and nonlinear (Rutherford 1973; Waelbroeck 1989) regime. Below, we briefly summarize the basic properties of the instability, obtained from linear analysis in the resistive MHD framework. We assume, as usually done, an incompressible, homogeneous density plasma.

Tearing modes are long-wavelength modes, that is, unstable perturbations have wavelengths larger than the shear length (or thickness) $a$ of the equilibrium magnetic field $\boldsymbol{B}$. Such unstable modes lead to the growth of magnetic islands via reconnection at the magnetic neutral line, or, more generally, on 'resonant' surfaces where $\boldsymbol{k} \cdot \boldsymbol{B}=0$, $\boldsymbol{k}$ being the wave vector of the perturbation along the sheet (figure 2). Instability grows on a time scale $\sim 1 / \gamma$ that is intermediate between the dissipative time of the equilibrium magnetic field, $\tau_{\eta}=a^{2} / \eta$, and the Alfvén crossing time $\bar{\tau}=a / v_{A}$ based on the thickness $a$. It is worth noting that since MHD does not have any intrinsic scale, lengths are traditionally normalized to the thickness $a$ of the sheet - differently 
(a)

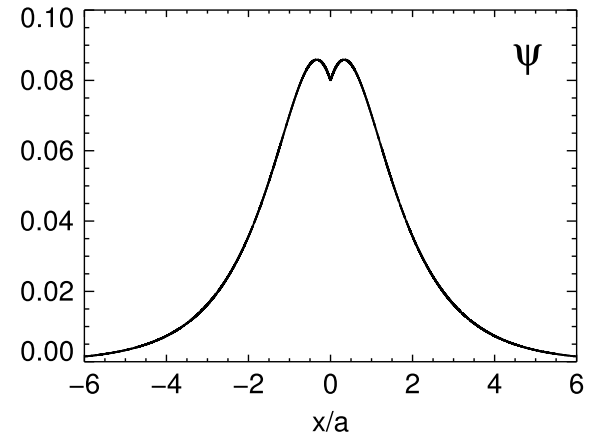

(b)

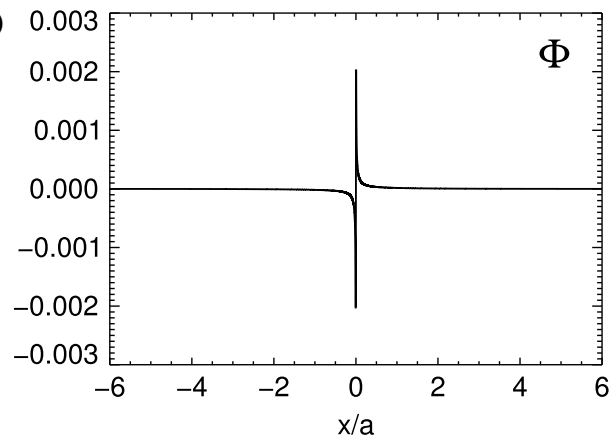

FIGURE 3. Tearing eigenfunctions for $S=10^{6}$ and $k a=0.8$ (small $\Delta^{\prime}$ ): magnetic field flux function $\psi(a)$ and velocity field streamfunction $\phi(b)$.

from the SP model in which, instead, $L$ is the macroscopic length - whereas time is normalized to $\bar{\tau}$. For the sake of clarity, we therefore label with an overbar the quantities defined through the current sheet thickness, $\bar{\tau}=a / v_{A}$ and $\bar{S}=a v_{A} / \eta$.

Since resistivity is negligible (or rather $\bar{S}^{-1} \ll 1$ ) everywhere except close to the resonant surface, tearing modes exhibit a quasi-singular behaviour in a small boundary layer of thickness $\delta$, the inner diffusion region, in which the perturbed magnetic and velocity fields exhibit sharp gradients, and where reconnection can take place. Out of the inner region, where resistivity can be neglected, perturbations smoothly decay to zero far from the resonant surface. An example of the eigenfunctions of the magnetic flux and velocity streamfunctions $\psi$ and $\phi$ at $\bar{S}=10^{6}$ is shown in figure 3 . In this case, the plotted eigenfunctions correspond to a Harris current sheet equilibrium, which is the one considered most in the literature and has a magnetic field profile $\boldsymbol{B}=B_{0} \tanh (x / a) \hat{\boldsymbol{y}}$. In perfectly antisymmetric equilibria, as the one chosen here, the eigenfunctions have a well-defined parity: the magnetic flux function is symmetric in $x$, being proportional to the reconnected magnetic field component at the neutral line, whereas the velocity streamfunction, proportional to the perturbed plasma velocity perpendicular to the sheet, is antisymmetric. As can be seen in figure $3(b)$, the plasma strongly accelerates while flowing into the inner layer, with the streamfunction ideally diverging as $\phi /\left(a v_{A}\right) \sim a / x$ for $|x / a| \rightarrow 0$. Resistivity becomes non-negligible close to the neutral line, regularizing the singularity: inside the inner layer, the plasma decelerates to the stagnation point, where it deflects outwards along the sheet, while the magnetic field diffuses and reconnects.

Instability is fed by the equilibrium current gradients which enter through the parameter $\Delta^{\prime}(k)$, or for brevity $\Delta^{\prime}$. The latter is defined as the discontinuity of the logarithmic derivative of the outer flux function when approaching the singular layer, and is a measure of the free energy of the system. The $\Delta^{\prime}$ parameter defines the instability threshold condition, instability occurring only when $\Delta^{\prime}>0$ (Furth et al. 1963; Adler, Kulsrud \& White 1980). For a Harris current sheet $\Delta^{\prime} a=2\left[(k a)^{-1}-k a\right]$, so that the unstable modes have wave vector satisfying $k a<1$.

The $\Delta^{\prime}$ parameter also controls the linear evolution. There are indeed two regimes that describe the unstable spectrum depending on the value of $\Delta^{\prime} \delta$. One is the so called constant- $\psi$ regime, traditionally referred to as tearing mode or small $\Delta^{\prime}$ regime (Furth et al. 1963). The other one is the non-constant- $\psi$ regime, or large $\Delta^{\prime}$, also known as resistive internal kink (Coppi et al. 1976). The difference between these two regimes is in the ordering of the derivatives of $\psi$ within the inner layer that leads 
to two different limiting cases of the dispersion relation (Ara, Basu \& Coppi 1978): in the small $\Delta^{\prime}$ regime $\psi^{\prime} \sim \psi \Delta^{\prime}$ and $\psi^{\prime \prime} \sim \psi \Delta^{\prime} \delta^{-1}$, that is, in the inner region $\psi$ is roughly constant and can be approximated by $\psi(0)$, provided $\Delta^{\prime} \delta \ll 1$; at larger values of $\Delta^{\prime}$ this approximation breaks down as $\psi$ has stronger gradients, $\psi^{\prime} \sim \psi \delta^{-1}$ and $\psi^{\prime \prime} \sim \psi \delta^{-2}$. In particular, the growth rate $\gamma$ and the inner layer $\delta$ scale in these two regimes as

$$
\begin{gathered}
\gamma \bar{\tau} \sim \bar{S}^{-3 / 5}(k a)^{2 / 5}\left(\Delta^{\prime} a\right)^{4 / 5}, \quad \frac{\delta}{a} \sim \bar{S}^{-2 / 5} \quad\left(\Delta^{\prime} \delta \ll 1\right), \\
\gamma \bar{\tau} \sim \bar{S}^{-1 / 3}(k a)^{2 / 3}, \quad \frac{\delta}{a} \sim \bar{S}^{-1 / 3} \quad\left(\Delta^{\prime} \delta \gg 1\right) .
\end{gathered}
$$

The expressions above can also be found as special cases of a general dispersion relation valid for any given value of the parameter $\Delta^{\prime}$ (Pegoraro \& Schep 1986).

Roughly speaking, the small and the large $\Delta^{\prime}$ regimes have wave vectors lying to the right and to the left of the fastest growing mode $k_{m}$, respectively. For example, in the case of a Harris sheet the two regimes correspond to a region in $k$-space $k_{m} a<k a<1$ in which the growth rate decreases with $k$ (small $\Delta^{\prime}$ ) and another one $0<k a<k_{m} a$ where the growth rate increases with $k$ (large $\Delta^{\prime}$ ). The scaling relations for the fastest growing mode can therefore be obtained by matching the two regimes (Bhattacharjee et al. 2009; Loureiro et al. 2013; Del Sarto et al. 2016). Since the expressions for $\gamma \bar{\tau}$ given in (2.1)-(2.2) should coincide at the fastest growing mode, the wave vector $k_{m}$ can be obtained by equating the right-hand side of the growth rate in the small and large $\Delta^{\prime}$ regimes, respectively. The scaling of the maximum growth rate $\gamma_{m}$ follows directly by either the small or the large $\Delta^{\prime}$ growth rate at $k=k_{m}$, leading to

$$
k_{m} a \sim \bar{S}^{-1 / 4}, \quad \gamma_{m} \bar{\tau} \sim \bar{S}^{-1 / 2}, \quad \frac{\delta_{m}}{a} \sim \bar{S}^{-1 / 4} \quad \text { (Fastest growing mode). } \quad(2.3 a-c)
$$

In figure 4 we show the transition from the small to the large $\Delta^{\prime}$ regime by plotting $\gamma \bar{\tau}$ as a function of $\bar{S}$ at two different wave vectors, $k a=0.01$ (light-blue dots) and $k a=0.05$ (red dots). The dashed lines correspond to the asymptotic scalings of the growth rate given in (2.1)-(2.3). As can be seen, as the Lundquist number $\bar{S}$ increases, the growth rate for a given wave vector moves from large to small $\Delta^{\prime}$ regimes. The transition is marked by a break in the slope of $\gamma \bar{\tau}$ when the given wave vector corresponds to the fastest growing mode for a specific pair of $\{\gamma \bar{\tau}, \bar{S}\}_{k=k_{m}}$, so that the envelope of the break-points for all $k$ values scales as $\bar{S}^{-1 / 2}$, as expected.

\section{Stability of thin current sheets and the 'ideal' tearing mode}

In the traditional theory of tearing mode the current sheet aspect ratio is fixed and its thickness $a$ is assumed to be macroscopic. On the other hand if a current sheet becomes thin enough, both the Alfvén time which normalizes the growth rate and the Lundquist number become smaller and smaller. Therefore, even if the growth rate appears to be small, it might actually be large when physically calculated in terms of macroscopic quantities. Indeed, as first seen by Biskamp (1986), the thin SP current sheet appeared to become unstable to fast reconnecting mode once a critical Lundquist number $S_{c}$ of order $10^{4}$ was passed. It is therefore of interest to consider generic current sheets whose aspect ratio scales with $S$ as $L / a \sim S^{\alpha}$, where now the scaling 


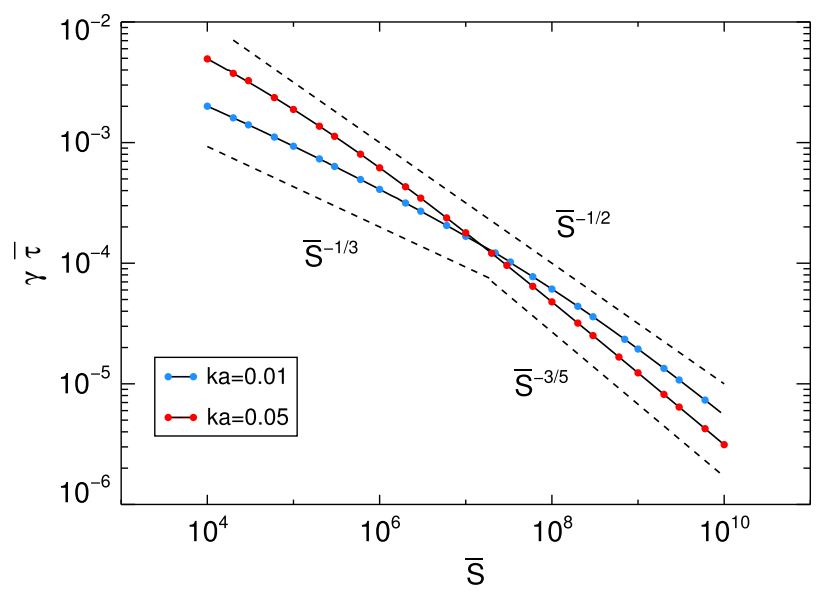

FIgURE 4. Growth rate $\gamma \bar{\tau}$ versus $\bar{S}$ : transition from the small to the large $\Delta^{\prime}$ regime for two different wave vectors. Dashed lines represent the asymptotic scalings of the large and small $\Delta^{\prime}$ regimes, $\gamma \bar{\tau} \sim \bar{S}^{-1 / 3}$ and $\gamma \bar{\tau} \sim \bar{S}^{-3 / 5}$, respectively, and of the fastest growing mode $\gamma \bar{\tau} \sim \bar{S}^{-1 / 2}$. The latter envelops the slope breaks occurring at the transition between small and large $\Delta^{\prime}$.

exponent $\alpha(\alpha=1 / 2$ for SP) is not specified (Bhattacharjee et al. 2009; Pucci \& Velli 2014). For the sake of simplicity, we assume that the background magnetic field corresponds to a Harris current sheet. Note that current sheets scaling as $L / a \sim S^{\alpha}$ diffuse on a time scale $\tau_{\eta}$ that is $\tau_{\eta} \sim \tau_{A} S^{1-2 \alpha}$. Plasma flows are therefore a necessary part of SP sheet equilibrium, which otherwise would diffuse in one Alfvén time. Indeed, the SP configuration is based precisely on the requirement that convective transport of magnetic flux balances Ohmic diffusion. Static equilibria can instead be constructed for thicker current sheets $(\alpha<1 / 2)$, as they diffuse over a time scale much longer than the ideal one, $\tau_{\eta} / \tau_{A} \gg 1$ for $S \gg 1$.

The main idea underlying 'ideal' tearing is the rescaling of the Lundquist number, which in the traditional tearing analysis is based on the thickness $a$ of the (macroscopic) current sheet equilibrium. If instead the length $L$ of the current sheet is considered as the macroscopic one, then the aspect ratio $L / a$ enters as a free parameter in the theory by introducing the renormalized quantities $\tau_{A}=\bar{\tau}(L / a)$ and $S=\bar{S}(L / a)$. This leads to a maximum growth rate of the tearing instability which increases with the aspect ratio:

$$
\gamma_{m} \tau_{A} \sim S^{-1 / 2}\left(\frac{L}{a}\right)^{3 / 2} \Rightarrow \gamma_{m} \tau_{A} \sim S^{-1 / 2+3 \alpha / 2} .
$$

Figure 5(a) shows the normalized maximum growth rate $\gamma_{m} \tau_{A}$ as a function of the inverse aspect ratio, that confirms the theoretical scaling given by (3.1).

Equation (3.1) shows that current sheets having an aspect ratio that scales as a power $\alpha$ of the Lundquist number $\alpha>1 / 3$ are tearing unstable with a maximum growth rate that diverges for $S \rightarrow \infty$. In particular, the growth rate of the plasmoid instability, $\gamma \tau_{A} \sim S^{1 / 4}$, is recovered for the scaling exponent of the SP sheet $\alpha=1 / 2$. This comes from the fact that in their original study Loureiro et al. (2007) neglect the effects of the equilibrium flows, therefore reducing the calculation to a standard 

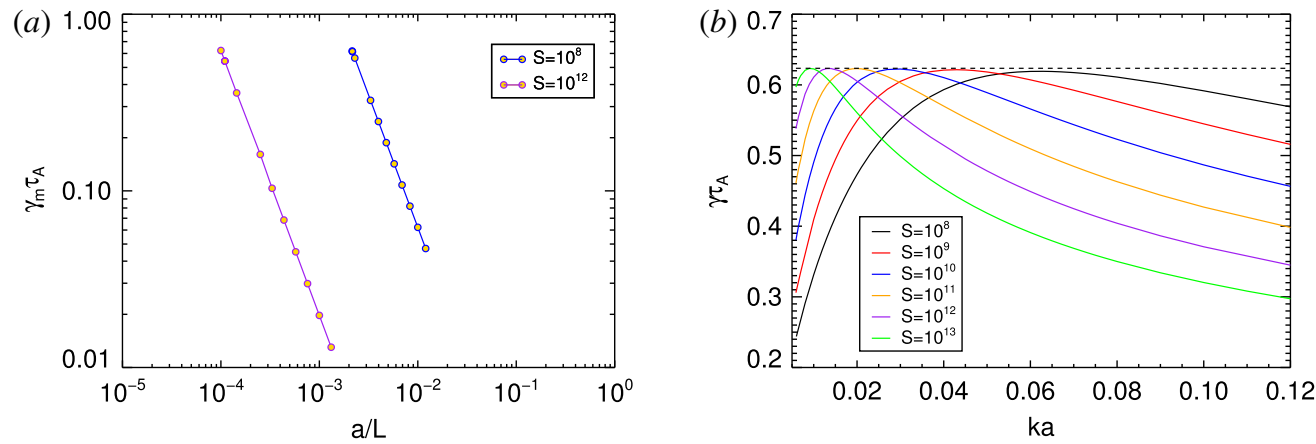

FIGURE 5. (a) Growth rates normalized to the Alfvén time as a function of the inverse aspect ratio. (b) Dispersion relations at $a / L=S^{-1 / 3}$ for different values of $S$ (from Pucci \& Velli 2014).

tearing mode boundary layer analysis (Loureiro et al. (2013) later found that flows seem to have negligible effect on the tearing mode within a SP sheet, although this result may be a consequence of the ordering assumptions). Current sheets at aspect ratios scaling with a power $\alpha<1 / 3$, on the contrary, are quasi-stable, i.e. $\gamma_{m}(\alpha<1 / 3) \rightarrow 0$ for $S \rightarrow \infty$. When instead $\alpha=1 / 3$, current sheets are 'ideally' unstable in the sense that the corresponding maximum growth rate becomes independent of $S$, and hence of order unity: as can be seen from figure $5(b)$, $\gamma_{m} \tau_{A} \rightarrow \gamma_{i} \tau_{A} \simeq 0.63$ for $S \rightarrow \infty$ (where the index ' $i$ ' stands for 'ideal'). As such, the aspect ratio $L / a \sim S^{1 / 3}$ provides an upper limit to current sheets that can naturally form in large Lundquist number plasmas, before they disrupt on the ideal time scale. Numerical simulations of a thinning current sheet, in which the thickness $a$ is parameterized in time, show that indeed the current sheet disrupts in a few Alfvén times via the onset of 'ideal' tearing, when the critical thickness is approached from above (Tenerani et al. 2015b), as we will discuss in more detail in $\S 4$.

To summarize, the ideally unstable current sheet has an inverse aspect ratio $a_{i} / L \sim S^{-1 / 3}$, with the wave vector and inner layer thickness of the fastest growing mode scaling with the Lundquist number as

$$
k_{i} L \sim S^{1 / 6}, \quad \frac{\delta_{i}}{L} \sim S^{-1 / 2} .
$$

An interesting point to remark is that the so-called inner diffusion or singular layer of the ideally unstable current sheet has an aspect ratio which scales with the Lundquist number in the same way as the SP sheet, $\delta_{i} / L \sim S^{-1 / 2}$. Yet, it is not a SP layer, because it does not correspond to a stationary equilibrium solution of the resistive MHD equations. The associated plasma flows into the X-points and along the inner sheet itself, $\tilde{u}_{\text {in }}$ and $\tilde{u}_{\text {out }}$, are increasing exponentially in time together with the reconnected flux, and their ratio does not follow the SP scaling but rather $\tilde{u}_{\text {in }} / \tilde{u}_{\text {out }} \sim S^{-1 / 3}$ : in this accelerating growing mode the ratio of inflow to outflow velocity is larger. The latter scaling property can be easily verified by exploiting the incompressibility condition $\tilde{u}_{\text {in }} / \delta_{i} \sim \tilde{u}_{\text {out }} k_{i}$ and (3.2). It is not a coincidence that the inner diffusion layer of the ideally tearing sheet scales like the SP, since the magnetic flux $\psi$ must now diffuse and reconnect on the ideal Alfvén time scale $\tau_{A}$ there, and the only length scale at which magnetic field diffuses on the Alfvén time is precisely 
$\delta_{i} / L \sim S^{-1 / 2}$. Stated in another way, within the inner layer we can approximate the diffusive term in Faraday's equation with $\eta \psi^{\prime \prime} \sim \eta \delta^{-2} \psi=\left(S \tau_{A}\right)^{-1}\left(L / \delta_{i}\right)^{2} \psi$, which must balance the growth rate $\gamma \psi \sim \tau_{A}^{-1} \psi$, yielding $\delta_{i} / L \sim S^{-1 / 2}$.

Although 'ideal' tearing has been considered here in the simpler case of resistive MHD, it provides a general framework for understanding under which conditions a fast tearing mode can develop. 'Ideal' tearing can be extended to include other physical effects which impact unstable current sheets, some of which are discussed below.

\subsection{Effects of plasma flows on current sheet stability}

The tearing mode within thin current sheets has been considered assuming static equilibria, even though current sheets form together with plasma flows into (inflows, $u_{\text {in }}$ ) and along (outflows, $u_{\text {out }}$ ) the sheet itself. As we have discussed, if $\alpha<1 / 2$ static equilibria can however be constructed that do not diffuse, or rather that diffuse over a time scale $\tau_{\eta}$ much longer than the ideal time scale. More precisely, inflows have little effect on the tearing mode if the diffusion rate within the inner reconnective layer, $u_{i n} / \delta$, is negligible with respect to the growth rate (Dobrott, Prager \& Taylor 1977), which is indeed the case for the ideal mode.

While equilibrium inflows can be neglected at large values of $S$, previous works (Bulanov et al. 1978; Bulanov, Sakai \& Syrovatskii 1979) showed that the inhomogeneous outflow along the sheet has instead a stabilizing effect on the tearing mode: outflows may therefore induce the formation of thinner sheets having an inverse aspect ratio $a_{i} / L \sim S^{-\alpha_{c}}$, with $\alpha_{c}>1 / 3$.

As discussed heuristically in Biskamp (1986), the tearing mode is stabilized when the outflow rate $\Gamma_{0} \sim u_{\text {out }} / L \simeq v_{A} / L$ exceeds a fraction of the growth rate, $\Gamma_{0}>f \gamma_{m}$, and this could explain the empirical critical Lundquist number $S_{c} \simeq 10^{4}$ for the onset of plasmoid instability within sheets having an aspect ratio scaling as SP. The factor $f \simeq 0.5$ takes into account that growth rates deviate from their asymptotic values at low $S$. It is possible to extend this argument in order to obtain the scaling exponent $\alpha_{c}$ for 'ideal' tearing in current sheets with outflows (M. Velli, Private communication 2015; Tenerani et al. 2015b). Since $\gamma_{m} \tau_{A}=\gamma_{i} \tau_{A} S^{-1 / 2+3 \alpha_{c} / 2}$, where $\gamma_{i} \tau_{A} \simeq 0.63$, then the condition for an $S$-independent growth rate is given by $\Gamma_{0}=f \gamma_{i} S^{-1 / 2+3 \alpha_{c} / 2}$, that leads to

$$
\alpha_{c}=\frac{2 \log \mu+\log S}{3 \log S} .
$$

In (3.3), $\mu=\Gamma_{0} /\left(f \gamma_{i}\right)$ and in particular the value $\mu=10$ (Biskamp 1986) yields the observed $\alpha_{c}=1 / 2$ for $S=10^{4}$, while, as expected, $\lim _{S \rightarrow \infty} \alpha_{c}=1 / 3$.

The impact of flows on the critical aspect ratio, expressed by (3.3), is consistent with recent numerical results (Tenerani et al. 2015b). Nevertheless, a more rigorous analysis of the effects of inflow-outflows on the stability of current sheets is still lacking, and a satisfactory explanation of the empirical stability threshold at low Lundquist numbers, $S \leqslant 10^{4}$, and its possible dependence on initial/boundary conditions, remains to be given. Note that outflows should also impact the number of islands which develop in any given simulation close to the stability threshold.

\subsection{Impact of viscosity on the critical aspect ratio}

Viscosity is relevant in many astrophysical environments (e.g. the interstellar medium), in the laboratory, and often in numerical simulations. The question therefore naturally arises as to how viscosity may impact the 'ideally' unstable current sheets. 


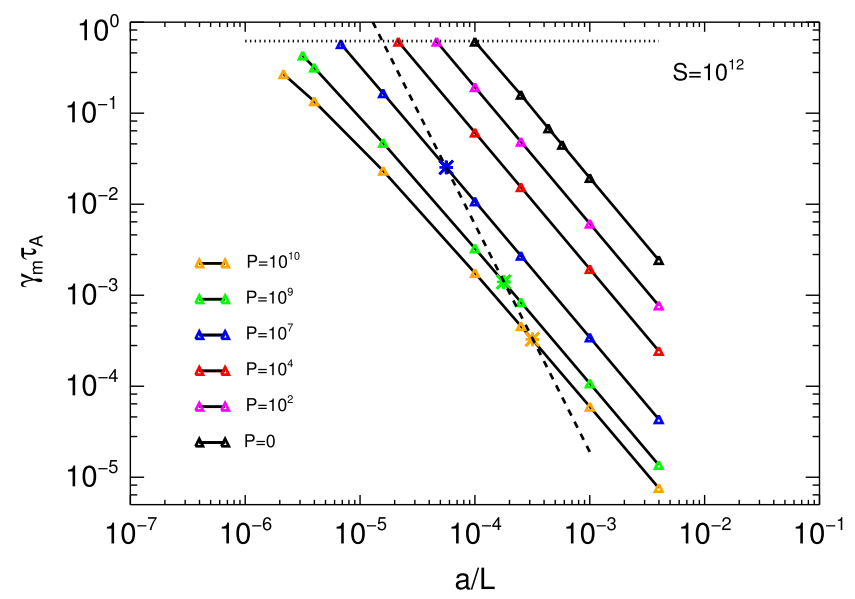

FIGURE 6. Growth rate normalized to the Alfvén time versus inverse aspect ratio at different Prandtl numbers. Stars correspond to the inverse aspect ratio of the viscous Sweet-Parker (from Tenerani et al. 2015a).

Effects of perpendicular viscosity on tearing modes in both the constant- $\psi$ and non-constant- $\psi$ regimes have been addressed by many authors in the past, showing that viscosity reduces the tearing mode growth rate (Bondeson \& Sobel 1984; Porcelli 1987; Grasso et al. 2008; Militello et al. 2011). As a consequence, more elongated current sheets can form in a way similar, in some sense, to what happens in the presence of outflows. Tenerani et al. (2015a) have shown that for large Prandtl numbers $P=v / \eta \gg 1$ ( $v$ is the perpendicular kinematic viscosity) the fastest growing mode has a growth rate that scales with $S, P$ and the aspect ratio $L / a$ as

$$
\gamma_{m} \tau_{A} \sim S^{-1 / 2} P^{-1 / 4}\left(\frac{L}{a}\right)^{3 / 2} \quad(P \gg 1),
$$

while the same scaling given by (3.1) holds for $P<1$. In figure 6 we plot some values of the maximum growth rate as a function of the inverse aspect ratio at different Prandtl numbers, for $S=10^{12}$. From (3.4) it is now possible to infer the scaling with $S$ and $P$ of the critical inverse aspect ratio, i.e. the one corresponding to an 'ideal' growth rate. In this case, the 'ideally' unstable current sheet is thinner by a factor $P^{-1 / 6}$ than the one in a non-viscous plasma, and is given by

$$
\frac{a_{i}}{L} \sim S^{-1 / 3} P^{-1 / 6} \quad(P \gg 1) \text {. }
$$

We conclude by noting that the Sweet-Parker current sheet in the presence of viscosity is instead thicker with respect to the inviscid case, $a_{S P} / L=S^{-1 / 2}(1+P)^{1 / 4}$ (Park, Monticello \& White 1984), whose inverse aspect ratio is indicated by the coloured stars in figure 6. Therefore, for large Prandtl numbers, $P \geqslant S^{2 / 5}$, the SP sheet can be quasi-stable to the tearing mode.

\subsection{Collisionless reconnection in the ideal regime}

In low-collision regimes where resistivity is effectively negligible, the dominant effects violating the ideal Ohm's law are electron inertia and/or anisotropic electron pressure 
tensors. While both can allow magnetic reconnection, the latter usually requires a kinetic model (Cai \& Lee 1997; Scudder et al. 2014), so the electron skin depth $d_{e} \equiv c / \omega_{p e} \propto \sqrt{m_{e}}$ often appears as the only non-ideal term driving collisionless tearing modes when a fluid description of the plasma is adopted. In this case, the small parameter is not $S^{-1}$ but rather the normalized electron skin depth $d_{e} / L$.

As a first step, we have considered the regime in which magnetic reconnection is induced by electron inertia in the strong guide field limit. The latter can be described by the Reduced MHD model in which the Hall term in Ohm's law can be neglected to the lowest order (Strauss 1976; Schep, Pegoraro \& Kuvshinov 1994; Del Sarto, Califano \& Pegoraro 2006). This description is suited, for instance, for tokamak and magnetically confined plasmas at low $\beta$, where $\beta=8 \pi P / B^{2}$ is the thermal to magnetic pressure ratio. It has been shown by Del Sarto et al. (2016) that in this regime the wave vector and growth rate of the fastest growing mode scale with the system parameters as

$$
k_{m} a \sim \frac{d_{e}}{a}, \quad \gamma_{m} \tau_{A} \sim\left(\frac{d_{e}}{L}\right)^{2}\left(\frac{L}{a}\right)^{3}, \quad \frac{\delta_{m}}{a} \sim \frac{d_{e}}{a} .
$$

From the scaling of the maximum growth rate $\gamma_{m} \tau_{A}$ in (3.6) it follows that onset of 'ideal' reconnection, that is when the growth rate becomes independent of the small non-ideal parameter $d_{e} / L$, occurs once a critical aspect ratio

$$
\frac{a_{i}}{L} \sim\left(\frac{d_{e}}{L}\right)^{2 / 3}
$$

is reached. In the limit of strong guide field it is possible to retain also some kinetic corrections related to electron temperature anisotropies and ion finite-Larmor-radius (FLR) effects in a simple way (Schep et al. 1994; Waelbroeck, Hazeltine \& Morrison 2009), that can be included in the reduced MHD model when approximated to their dominant gyrotropic contribution (we then speak of gyrofluid models). These models can be used also at relatively large $\beta$ values (Grasso, Tassi \& Waelbroeck 2010). Electron temperature effects arise at length scales of the order of the ion sound Larmor radius $\rho_{s}=c_{s} / \Omega_{i}, c_{s}=\sqrt{T_{e} / m_{i}}$ being the ion sound speed and $\Omega_{i}$ the ion cyclotron frequency. These additional terms in the generalized Ohm's law can be seen as the result of an anisotropic electron pressure contribution (Schep et al. 1994), or as the Hall term contribution when the diamagnetic drift is retained as a first-order correction to the $\boldsymbol{E} \times \boldsymbol{B}$ electron drift (see also Del Sarto et al. 2016). Ion FLR effects introduce as a further length scale the ion Larmor radius $\rho_{i}=v_{t h}^{i} / \Omega_{i}$, where $v_{t h}^{i}$ is the ion thermal speed. Depending on the way ion FLR are approximated from kinetic theory, different gyrofluid models can be obtained, all yielding the same dispersion relation for finite temperature tearing instabilities when $\rho_{\tau}^{2} \equiv \rho_{s}^{2}+\rho_{i}^{2} \gg d_{e}^{2}$ (Pegoraro \& Schep 1986; Porcelli 1991; Ottaviani \& Porcelli 1995). An explicit expression for the fastest growing mode valid in this regime can be found in Comisso et al. (2013). For a Harris sheet, when rescaled from the sheet thickness $a$ to the length $L$, the wavenumber and growth rate of the fastest growing mode are therefore the following (Del Sarto et al. 2016),

$$
k_{m} a \sim \frac{d_{e}}{L}\left(\frac{\rho_{\tau}}{d_{e}}\right)^{1 / 3} \frac{L}{a}, \quad \gamma_{m} \tau_{A} \sim\left(\frac{d_{e}}{L}\right)^{2} \frac{\rho_{\tau}}{d_{e}}\left(\frac{L}{a}\right)^{3} \quad\left(\rho_{\tau} \gg d_{e}\right) .
$$


In this regime, the critical inverse aspect ratio scales with both $d_{e} / L$ and $\rho_{\tau} / d_{e}$ as

$$
\frac{a_{i}}{L} \sim\left(\frac{d_{e}}{L}\right)^{2 / 3}\left(\frac{\rho_{\tau}}{d_{e}}\right)^{1 / 3} \quad\left(\rho_{\tau} \gg d_{e}\right) .
$$

In conclusion, kinetic effects increase the linear growth rate by a factor $\rho_{\tau} / d_{e}$ with respect to the low- $\beta$ case, as can be seen by comparing the growth rate in (3.6) with the one including finite temperature effects in (3.8). As a consequence, the critical inverse aspect ratio turns out to be thicker in the latter case by a factor scaling as $\left(\rho_{\tau} / d_{e}\right)^{1 / 3}$ (cf. (3.7) and (3.9)).

\section{The trigger problem}

Observations show that many explosive phenomena usually display an initial phase (of long but uncertain duration) during which energy is transferred to and accumulated in the system in question, followed by an abrupt destabilization process expressed by an impulsive phase, often attributed to the onset, or trigger, of magnetic reconnection. During the impulsive phase energy is suddenly released on a fast (ideal) time scale (of the order of a few $\tau_{A}$ ) in the form of heat, kinetic energy and populations of accelerated particles. The impulsive phase is followed by main and recovery phases, whereby energy released becomes more gradual and the system relaxes towards a quiet configuration (Akasofu 1964; Wesson 1986; Fletcher et al. 2011). Solar flares provide one of the most spectacular examples in this sense: a prominence can stand for weeks in the solar corona until it erupts by releasing a huge amount of energy (the flare), of about $10^{30}-10^{32} \mathrm{erg}$, in a few tens of minutes or hours, depending on their size. The flare displays an impulsive phase, that can be seen as sudden intensity enhancements in different wavelengths, especially in the hard X-ray spectrum, lasting usually no more than a few minutes for the larger events (Ellison 1946; Kane 1974; Ajello et al. 2014).

In the weakly collisional plasmas found in many astrophysical environments, or in fusion devices, bulk plasma motions tend to form thin current sheets spontaneously in localized regions (Syrovatskiir 1971; Biskamp 1993). There, the intense currents are limited only by the extremely small resistivity or by other effects, such as electron inertia, until they ultimately relax once reconnection is enabled inside these thin boundary layers. The problem of understanding in which way thin current sheets form is a complex and rich one, both theoretically and numerically. We therefore do not go into details, but some recent studies about thin current sheet formation for configurations relevant to magnetosphere dynamics and the solar corona can be found for instance in Birn \& Schindler (2002), Titov, Galsgaard \& Neukirch (2003), Aulanier, Pariat \& Démoulin (2005), Rappazzo \& Parker (2013), Hsieh \& Otto (2015).

Whatever the mechanism might be, one can always imagine that, during current sheet formation, the system evolves through a sequence of similar configurations to which the linear stability analysis can be applied in order to investigate its properties. As shown in $\S 3$, the growth rate of the reconnecting tearing mode instability exhibits a strong dependence on the current sheet aspect ratio, for example in resistive MHD $\gamma \tau_{A} \propto(L / a)^{3 / 2}$ (cf. (3.1)). This, together with the large values of the Lundquist number, or $L / d_{e}$, suggests that the formation of small scales all the way down to the critical thickness would naturally lead to the trigger of fast reconnection. 


\subsection{Scenario}

In the following, we will assume a current profile that thins exponentially in time. Exponentially thinning current sheets are indeed of particular importance, as they are commonly observed in simulations of solar and stellar coronal heating (Rappazzo \& Parker 2013), as self-similar solutions of X-point collapse (Sulem et al. 1985), as well as in in situ measurements during the growth phase of substorms in the Earth's magnetotail (Sanny et al. 1994), to give some examples.

We therefore assume that the ambient plasma and magnetic fields evolve over a time scale $\tau_{c}$ which is determined by ideal plasma motions and hence does not depend on $S$. Consider the simpler case of resistive (incompressible) MHD, and take a Harris current sheet of inverse aspect ratio $a / L$ shrinking on a time scale $\tau_{c} \sim a(\mathrm{~d} a / \mathrm{d} t)^{-1} \gtrsim \tau_{A}$. The parameter $\tau_{c}$ thus represents schematically the coupling between the local dynamics within the current sheet and some external process inducing the thinning itself. Within this framework, we extend the tearing mode analysis to systems that are parameterized in time through the evolving aspect ratio (Tenerani et al. 2015b).

An investigation of the disruption of a forming current sheet germane to the present discussion has been recently published in Uzdensky \& Loureiro (2016). Since the two scenarios differ somewhat, let us briefly comment on the differences here, although we refer to that paper for more details. Our study focuses on the linear stage of the tearing instability to provide a possible explanation for the onset of fast reconnection, using a WKB approach to describe the growth of unstable modes during the dynamical collapse of the sheet. In this way, we take into account that unstable modes with a growth rate exceeding $\tau_{c}^{-1}$ can in principle compete in disrupting the current sheet. Uzdensky \& Loureiro (2016), on the other hand, consider arbitrary time scales for driving the sheet collapse, and start from the assumption that the linear stage of a mode ends once its growth rate exceeds the driving rate. They then estimate that the first mode to meet this condition will also be the one dominating the subsequent nonlinear evolution. They therefore examine the nonlinear regime to describe the disruption of the current sheet, which in their scenario may occur also on time scales scaling as a positive power of $S$. In this sense, they do not impose restrictions on mode growth times with respect to dissipative coefficients. Our approach on the other hand focuses precisely on the latter aspect, our framework being one in which things will occur on the fastest possible times compatible with the dynamical system in question, and therefore, in analogy for example with turbulence, we expect the fastest growing modes not to scale with the dissipative coefficients. So in some sense the approach of Uzdensky \& Loureiro (2016) is more general than ours, but does not investigate in detail whether and under which conditions the transition to fast reconnection (the trigger) might occur. Other recent interesting analyses of a similar conceptual reconnection problem, the Taylor's problem, have been provided by Comisso, Grasso \& Waelbroeck (2015) and Vekstein \& Kusano (2015). They analyse the possible time evolution of an initial macroscopic, stable current layer which is subject to a finite-amplitude perturbation at its boundaries, and recognize the role sufficiently fast tearing modes may play in disrupting the current sheet. We do not discuss that problem here, although it would be interesting to study how in the asymptotic limit of large $S$ the 'ideal' tearing framework affects those models. Let us focus now on our scenario of an exponentially thinning sheet.

On the basis of the rescaling argument, the growth rates in the small and large $\Delta^{\prime}$ regimes discussed in $\S 2$ can now be generalized to arbitrary aspect ratios. By taking $\Delta^{\prime} a \sim\left[(k a)^{-1}-k a\right]$ for a Harris sheet, these are given, respectively, by

$$
\gamma \tau_{A} \sim S^{-3 / 5}\left(\frac{a}{L}\right)^{-8 / 5}(k a)^{-2 / 5}\left[1-(k a)^{2}\right]^{4 / 5}, \quad \gamma \tau_{A} \sim S^{-1 / 3}\left(\frac{a}{L}\right)^{-2}(k a)^{2 / 3} .
$$


The fastest growing mode, as already shown in $\S 3$, has the growth rate scaling as

$$
\gamma_{m} \tau_{A} \sim S^{-1 / 2}\left(\frac{a}{L}\right)^{-3 / 2}
$$

whereas the wave vector and thickness of inner diffusion layer scale, respectively, as

$$
k_{m} a \sim S^{-1 / 4}\left(\frac{a}{L}\right)^{-1 / 4}, \quad \frac{\delta_{m}}{a} \sim S^{-1 / 4}\left(\frac{a}{L}\right)^{-1 / 4} .
$$

As the thinning proceeds in time, modes with increasing wave vector $k$ are progressively destabilized, owing to the traditional instability condition $k a(t)<1$ in a Harris sheet. In this way, each newly destabilized mode lies in the small $\Delta^{\prime}$ regime and it transitions toward the large $\Delta^{\prime}$ one as time elapses, by crossing at some point the fastest growing mode. This can be understood also by looking back at the plot in figure 4: since $\bar{S} \equiv S(a / L)$, if $a / L$ decreases at fixed $S$, then $\bar{S}$ decreases accordingly, so that a given mode shifts from the right to left side of the plot. On the other hand, only those modes fitting within the current sheet, that therefore satisfy $k L \geqslant 2 \pi$, can grow. This implies that at the beginning, when $a \lesssim L$, there are only unstable modes in the small $\Delta^{\prime}$ regime. This initial stage in turn ends when the smallest unstable mode, which has a wavelength of the order of the current sheet length $L, k \sim 2 \pi / L$, coincides with $k_{m}$ (Uzdensky \& Loureiro 2016). This happens when $S^{-1 / 4}(a / L)^{-1 / 4} \simeq 2 \pi(a / L)$, or, in other words, when $a / L \lesssim 0.2 S^{-1 / 5}$. At that point, the fastest growing mode will be always unstable, and hence it will dominate over both the small and the large $\Delta^{\prime}$ modes. The initial stage evolving in the small $\Delta^{\prime}$ regime however does not contribute significantly to the growth of perturbations so one can consider only the regime given by the fastest growing mode, expressed by (4.2)-(4.3), to describe the transition to fast reconnection. Indeed, the small $\Delta^{\prime}$ (as well as the large $\Delta^{\prime}$ ) regime has a growth rate that, even if the current sheet is thinning, goes to zero for $S \rightarrow \infty$ (according to (4.1)). As a consequence, modes in that regime cannot grow during a finite interval of time of order of $\tau_{c}$. In other words, the small $\Delta^{\prime}$ regime corresponds to slow reconnection and it cannot in general provide the transition to fast reconnection in quasi-ideal plasmas. Assuming a collapse of the form $a(t)=\exp \left(-t / \tau_{c}\right)$, which can be easily generalized to include an exponential increase of the length $L$, then the amplitude of the reconnecting magnetic flux $\psi$ is given by

$$
\psi(t)=\psi(0) \exp \left(\int_{0}^{t} \gamma\left(t^{\prime}\right) \mathrm{d} t^{\prime}\right)
$$

where, by neglecting the initial small $\Delta^{\prime}$ stage (current sheets having $a / L>S^{-1 / 5}$ ), the integrand is given by

$$
\gamma(t)=\gamma_{m}(t)=\frac{1}{\tau_{A}} 0.63 S^{-1 / 2}\left[\exp \left(\frac{t}{\tau_{c}}\right)\right]^{3 / 2} .
$$

Expressions (4.4)-(4.5) yield

$$
\psi(t)=\psi(0) \exp \left[\frac{2 \tau_{c}}{3} 0.63 S^{-1 / 2}\left(\mathrm{e}^{3 t /\left(2 \tau_{c}\right)}-1\right)\right],
$$

that can be approximated as follows,

$$
\begin{gathered}
\psi(t) \simeq \psi(0) \mathrm{e}^{\gamma_{m}(0) t}, \quad \text { if } t \ll \tau_{c} 2 / 3, \\
\psi(t) \simeq \psi(0) \mathrm{e}^{\gamma_{m}(t) 2 \tau_{c} / 3}, \quad \text { if } t>\tau_{c} 2 / 3
\end{gathered}
$$



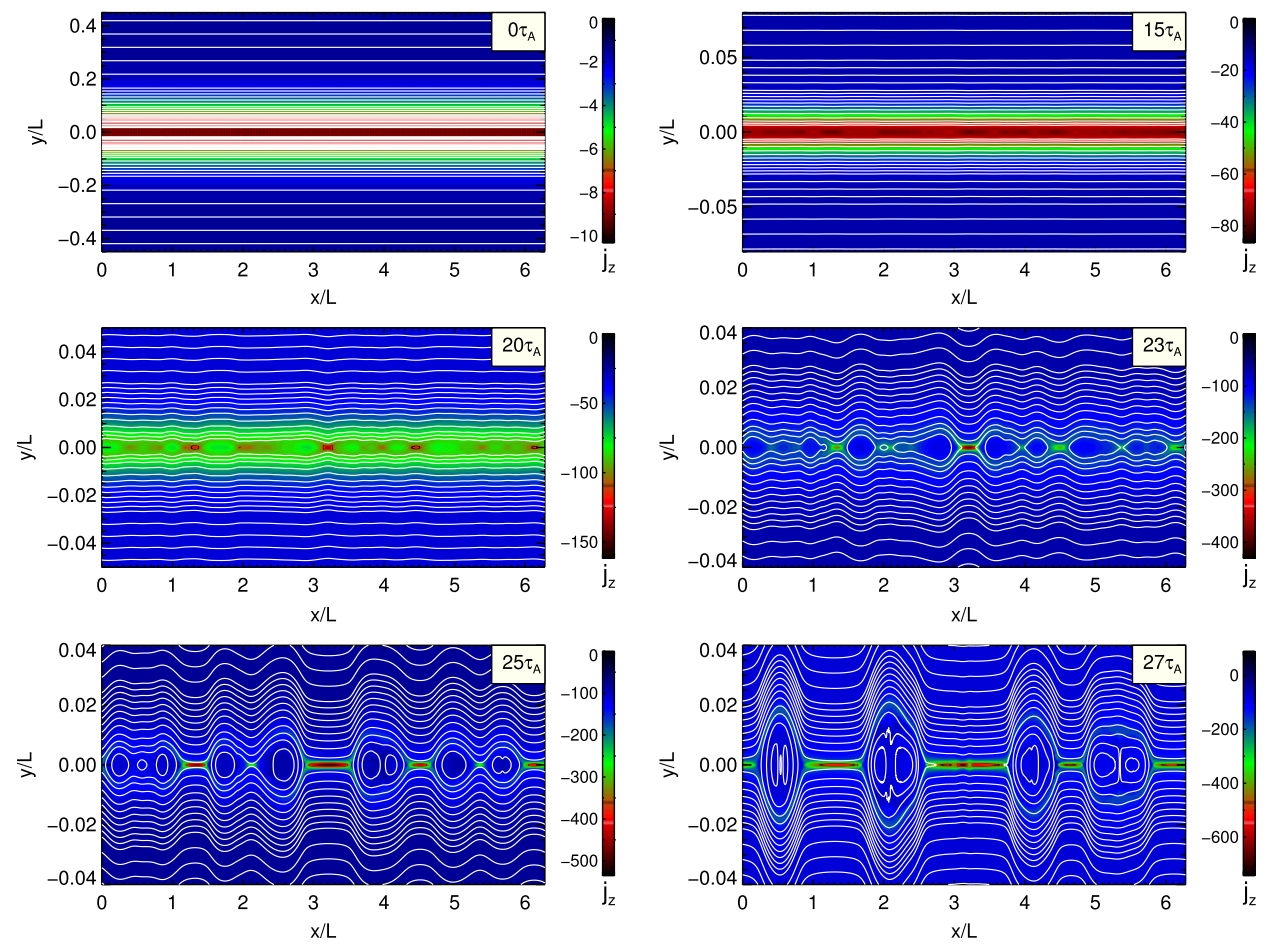

FIgURE 7. Temporal evolution of a thinning Harris current sheet. The out-of-plane current density $j_{z}$ is colour coded and white lines represent magnetic field lines.

The onset of the tearing instability roughly takes place when unstable modes have time to develop, hence at about the time $\tau_{*}$ such that $\gamma_{m}\left(\tau_{*}\right) \tau_{c} \simeq 1$, as can be seen also from (4.8). In turn, the growth rate rapidly increases while approaching the critical thickness $a_{i} / L \sim S^{-1 / 3}$ from above, so one can easily be convinced that a fast current sheet collapse naturally drives an explosive transition from a quasi-stable state - growth rate depending on a negative power of $S$ - to an ideally unstable one.

We have tested such scenario with fully nonlinear MHD simulations in which a collapse of the current sheet is imposed a priori with $\tau_{c}$ in the range $1-10 \tau_{A}$ (Tenerani et al. 2015b). Figure 7 shows an example of the temporal evolution of an exponentially thinning Harris sheet initially perturbed by a random noise of fluctuations. The Lundquist number in these simulations is set to $S=10^{6}$ and the Prandtl number to $P=1$, so that the scalings of the non-viscous case are not modified significantly. The in-plane magnetic field is $\boldsymbol{B}=B_{0} \tanh [y / a(t)] \hat{\boldsymbol{x}}$ and, for numerical convenience, a thinning of the form $a(t)=a_{0} \exp \left(-t / \tau_{c}\right)+a_{\infty}\left(1-\exp \left(-t / \tau_{c}\right)\right)$ has been chosen. For the case shown in figure 7 we have fixed $a_{\infty} / a_{0}=0.1$, with $a_{0}=0.1 L$ and $\tau_{c}=4 \tau_{A}$. In figure 8 we show the temporal evolution of $a(t)(a)$ and of some unstable Fourier modes of $\psi$ at the neutral line $y=0(b)$ for the same simulation. The simulation illustrates that during the linear stage of the instability (from $t \simeq 11$ to $t \simeq 22 \tau_{A}$ ) the magnetic field rapidly reconnects in a few Alfvén times when the critical thickness $a_{i} / L \sim S^{-1 / 3} \simeq 0.01$ is approached from above, and that the current breaks up into a number of magnetic islands $N_{i}$ that scales as $N_{i} \sim S^{1 / 6} \simeq 10$. As can be seen from figure 7 , the following nonlinear stage is characterized by 


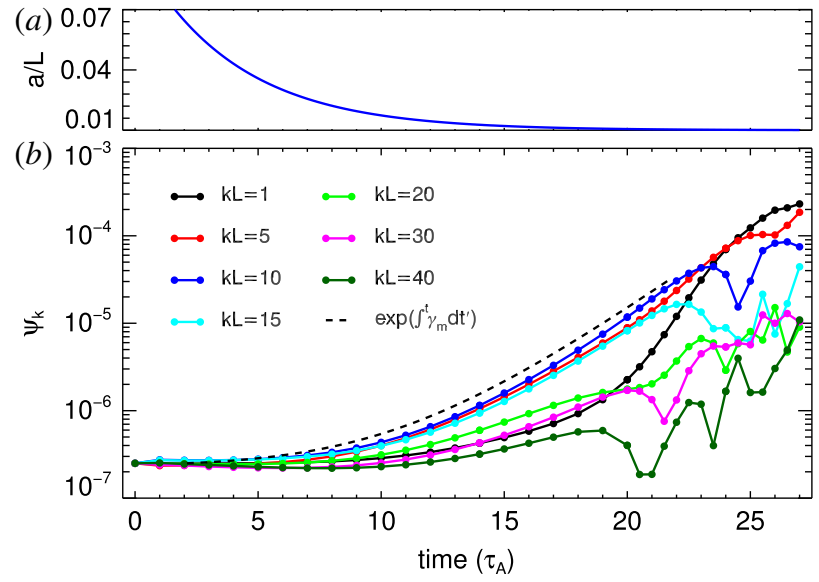

FIGURE 8. Temporal evolution of the current sheet thickness $(a)$ and of the amplitude of some Fourier modes of the flux function $\psi$ at the neutral line $(b)$ of the simulation shown in figure 7. The dashed line corresponds to the integral given in (4.4) where the functional form $a(t)$ adopted in the simulation (see text) has been used (from Tenerani et al. 2015b).

the competition between coalescence of magnetic islands (this can be seen also by looking at the increasing magnitude of long-wavelength modes, $k L=5$ and $k L=1$, in figure 8) and X-point collapse, in a way qualitatively similar to that discussed in Malara, Veltri \& Carbone (1992). We defer more detailed discussions about the nonlinear evolution of an 'ideally' reconnecting current sheet to $\S 5$.

To summarize, in the scenario proposed above the mechanism driving the collapse (that can be mapped in the developmental phase, or the storage phase) is not specified. The ambient current sheet thins on a time scale $\tau_{c}$ which is independent from $S$ and instability is let to freely evolve from an initial slow phase of reconnection, when the thickness is larger than critical, to an ideally fast one while approaching the critical inverse aspect ratio $a_{i} / L \sim S^{-1 / 3}$. Numerical simulations of a thinning current sheet with $\tau_{c} \simeq 1-10 \tau_{A}$ show that when approaching the critical inverse aspect ratio from above magnetic islands significantly grow from the initial noise up to the thickness of the inner layer of the reconnecting current sheet itself and beyond, during the nonlinear stage, on a time scale of the order of a few $\tau_{A}$. In the model that we have chosen of an exponential collapse the time needed to form the ideally unstable current sheet is about $\tau_{i} \simeq\left(\tau_{c} / 3\right) \ln (S)$, i.e. it depends very weakly (logarithmically) on $S$ and allows for a two time scale dynamics. For instance, if $\tau_{c}=1-10 \tau_{A}$ and $S=10^{12}-10^{14}$ then $\tau_{i} \simeq 10-100 \tau_{A}$ thus one or two orders of magnitude larger than the time expected for the onset and development of 'ideal' reconnection.

Nonlinearities become important when the half-width $w$ of magnetic islands, given approximately by $w \simeq 2 a \sqrt{\psi /\left(B_{0} a\right)}$ (see, e.g. Biskamp 2000 pp. 82-83), is of the order of the half-thickness of the inner diffusion region of the reconnecting sheet $\delta_{m}$, right equation (4.3). This condition is met when the perturbation has an amplitude scaling as $\psi_{n l} /\left(B_{0} L\right) \sim 0.25 S^{-1 / 2}(a / L)^{1 / 2}$. For a critical current sheet $a_{i} / L \sim S^{-1 / 3}$ this estimate yields a nonlinear amplitude $\psi_{n l, i} /\left(B_{0} L\right) \sim 0.25 S^{-2 / 3}$, and even smaller for thinner sheets. For example, the Sweet-Parker with $a_{S P} / L \sim S^{-1 / 2}$ has a nonlinear amplitude of about $\psi_{n l, S P} /\left(B_{0} L\right) \sim 0.25 S^{-3 / 4}$. In solar active regions $B_{0} \sim 100 \mathrm{G}$, $L \sim 10^{4} \mathrm{~km}$ and $S \simeq 10^{12}$, yielding $\psi_{n l} \sim 0.25 \times 10^{-9} \times\left(10^{4} \times 100\right)=0.25 \times 10^{-3} \mathrm{G} \mathrm{km}$ 


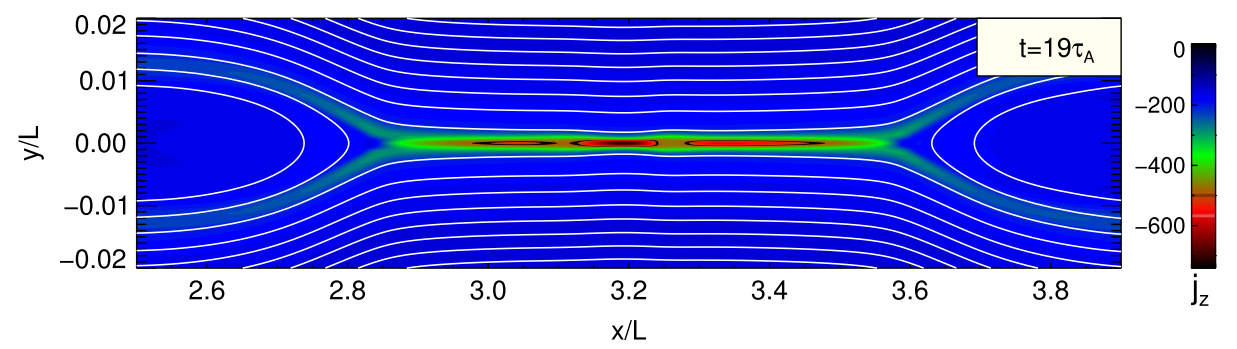

FIGURE 9. Blow up of the collapsing current sheet during the nonlinear stage of the primary ideal tearing instability (from Tenerani et al. 2015b).

with background magnetic flux of about $\psi_{0} \sim\left(B_{0} L\right) S^{-1 / 3} \sim 100 \mathrm{G} \mathrm{km}$, for $a / L \simeq S^{-1 / 3}$. Therefore, the initial perturbation should be about $0.25 \times 10^{-5}$ times smaller than the background. The duration of the linear phase depends on both the initial noise level and the growth rate of the instability (and it becomes shorter and shorter with $S$ for sheets thinner than critical). It is certainly clear that once the 'ideal' instability limit is approached the duration of the linear phase may be extremely limited. Nonetheless if the linear theory is thought of as providing a trigger for faster, nonlinear dynamics, as we will discuss in the next section, the 'ideally' unstable aspect ratio remains an upper limit for laminar current sheets. It is reasonable to expect that an initial nonlinear noise would lead to the disruption of the current sheet, and to a turbulent regime, on ideal time scales via the growth of resonant (most unstable) modes. In this regard a detailed study requires numerical simulations at different nonlinear noise levels in some sense generalizing the paper by Matthaeus \& Lamkin (1985).

\section{Recursive reconnection: the 'fractal' reconnection model revised}

$\mathrm{X}$-points arising from a tearing instability at the largest wavelengths, i.e. far from the small $\Delta^{\prime}$ regime, collapse into an elongated current sheet during the early nonlinear stage (Waelbroeck 1989; Jemella et al. 2003). In this respect, nonlinearities of the tearing mode provide a self-consistent mechanism for current sheet formation to which the scenario discussed in $\S 4$ can in turn be applied, on the basis of similarity and rescaling of length and time scales. This simple idea led Shibata \& Tanuma (2001) to propose a phenomenological 'fractal reconnection' model for flares, that we revisit here in light of the 'ideal' tearing scenario.

In general, it is observed that if the aspect ratio of the secondary current sheet becomes large enough, then the latter becomes unstable to secondary tearing generating secondary plasmoids (Malara et al. 1992; Loureiro et al. 2005). This can be seen in the last panel of figure 7 and in a blow-up of one of the secondary current sheets, displayed in figure 9, the latter obtained from a similar simulation with $S=10^{6}, P=1, \tau_{c}=\tau_{A}, a_{\infty} / a_{0}=0.1$ and $a_{0}=0.1 L$. A number of past numerical and theoretical studies have addressed the problem of the transition from the laminar reconnection, proper of the early nonlinear stage, to the subsequent highly unsteady one, characterized by intermittent generation of plasmoids within the sheet itself, bearing faster average reconnection rates (Lapenta 2008; Bhattacharjee et al. 2009). The Sweet-Parker paradigm has been constantly invoked in the interpretation of the nonlinear evolution - both to explain the instability onset within the laminar current sheet, and to model the following fully nonlinear plasmoid-dominated stage (Loureiro et al. 2005; Bhattacharjee et al. 2009; Cassak \& Drake 2009; Daughton et al. 2009; 
(a)

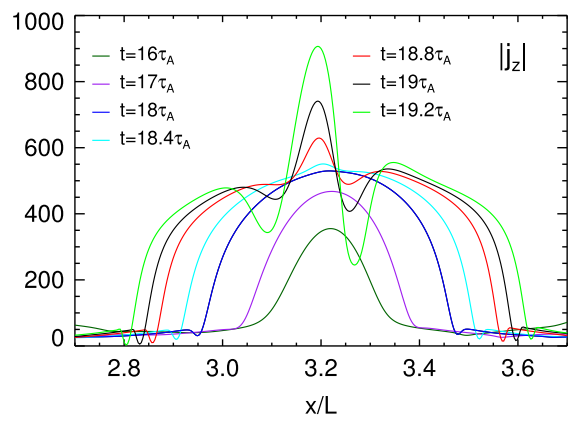

(b)

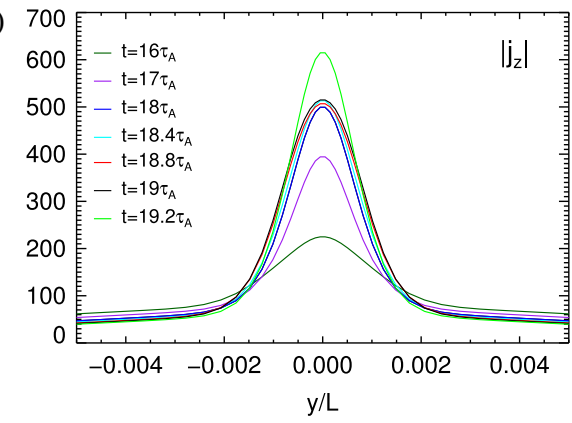

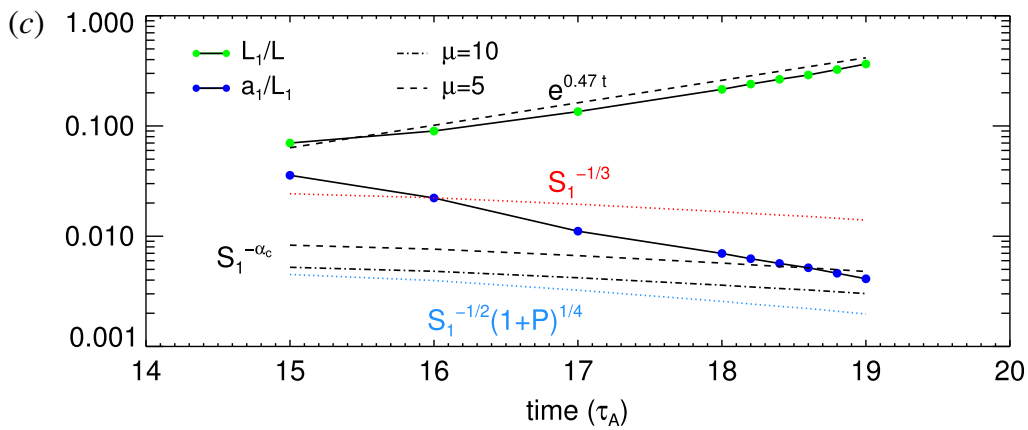

FIgURE 10. Details of the time evolution of the secondary current sheet shown in figure 9. $(a, b)$ Plot of the intensity of the out-of-plane current density $j_{z}$ along $((a)$, at $y / L=0)$ and across $((b)$, at $x / L=3.15)$ the sheet; the current half-length $L_{1}$ increases (exponentially) in time whereas its half-thickness $a_{1}$ is approximately constant, $a_{1} \simeq L 0.0015$. (c) (adapted from Tenerani et al. 2015b): time evolution of the inverse aspect ratio $a_{1} / L_{1}$ (blue dots) and of the length $L_{1} / L$ (green dots) of the sheet; we plot for reference the thresholds given by the 'ideal' tearing (red dotted line), the viscous Sweet-Parker (light blue dotted line), and the corrected-flow 'ideal' threshold (dashed and dot-dashed black lines), the latter according to (3.3).

Huang \& Bhattacharjee 2010; Uzdensky et al. 2010; Ali, Jiquan \& Kishimoto 2014). However, the onset of fast reconnection may take place at smaller aspect ratios for a given Lundquist number: in particular, 'ideal' tearing predicts a different instability criterion and different scaling laws for the dependence on (macroscopic) quantities $L$ and $S$, which, when the possible effects of flows are taken into account, may provide a better guide for inspecting the nonlinear evolution.

Recent numerical two-dimensional simulations of 'ideally' unstable sheets at Lundquist numbers $S=10^{6}-10^{7}$, have shown that the secondary current sheet lengthens exponentially at a rate close to the growth rate of the primary tearing (Tenerani et al. 2015b). This is seen in figure 10, where different panels describing the time evolution of the secondary current sheet shown in figure 9 are displayed. The (half) thickness and length of such a sheet is now labelled $a_{1}$ and $L_{1}$ : $(a)$ shows the profile of the out-of-plane current density $j_{z}(x, y)$ intensity along the current sheet at the neutral line $y / L=0$ at different times, ranging from the end of the linear stage of the primary tearing up to the fully developed secondary instability. The latter can be recognized as the growth of a more intense current maximum in the middle of $L_{1}$, surrounded by two local minima, corresponding to two plasmoids, at about $t=19.2 \tau_{A}$ (green colour); in $(b)$ we plot a cut of $j_{z}(x, y)$ across the sheet at $x / L=3.15$, at the 


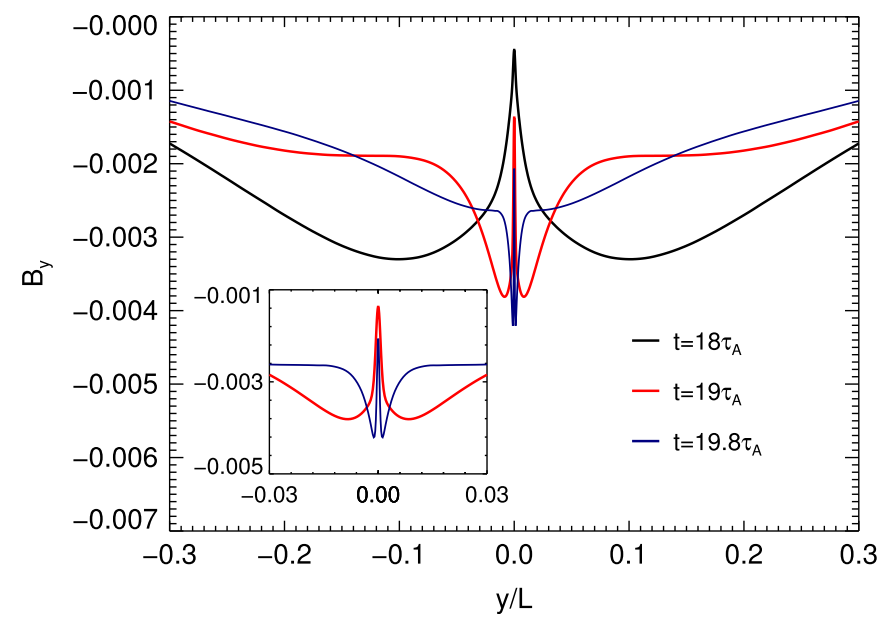

FIGURE 11. Hierarchy of tearing modes: plot of the magnetic field component along the inhomogeneous direction, $B_{y}$, versus $y / L$ at position $x / L=3.15$ of the current sheet shown in figure 9. Black colour corresponds to the primary tearing, the red one to $n=1$, and the blue to $n=2$. The inset is a blow up of the magnetic field for $n=1$ and $n=2$ (from Tenerani et al. 2015b).

same times. Such a secondary current sheet arises from the inner diffusion layer of the primary tearing: its thickness has indeed an almost constant value $a_{1} / L \simeq 0.0015$, that consistently scales with the macroscopic quantities as $a_{1} / L \simeq \delta_{i} / L \sim S^{-1 / 2}$ (see (3.2)). This can be seen from figure $10(b)$, for all times before the appearance of fully developed plasmoids (i.e. $t=19.2 \tau_{A}$ as mentioned above); in (c) we plot the time evolution of $L_{1}$ (green dots), $a_{1} / L_{1}$ (blue dots) and the threshold conditions for instability corresponding to the 'ideal' tearing (red dotted line), to the viscous SP (light blue dotted line), and to the flow-modified 'ideal' tearing discussed in $\S 3.1$ (black dashed and dot-dashed lines). As can be seen, this current sheet breaks up during the collapse once it becomes unstable on the local Alfvén time, hence before the SP aspect ratio is reached and in good agreement with the flow-modified 'ideal' tearing (Landi et al. 2015; Tenerani et al. 2015b), finally giving rise to another thinner sheet (see the green curves in figure $10(a, b)$. In figure 11 we show the profile of the magnetic field $B_{y}(y, x), x / L=3.15$, across the centre of the same sheet shown in figure 9 , at three different times. The magnetic field profile displays a striking if unsurprising similarity to the tearing eigenfunctions (compare also with figure $3 a$ ), as well as a hierarchical structure: the black colour corresponds to the magnetic perturbation grown during the primary instability; the red and blue colours correspond to the first and to the second secondary instability, magnified in the inset. In particular, note that the red profile as seen in the inset is the same as the black profile of the main figure. What this means is that each magnetic field perturbation that will lead to a new sheet grows within the inner diffusion layer of the unstable mode developed within the previous sheet. This in turn suggests that formation of ever smaller scales occurs via a recursive process of X-point formation, collapse and break up, reminiscent of the so-called 'fractal' reconnection scenario originally proposed by Shibata \& Tanuma (2001), that can be accounted for by the 'ideal' tearing instability. 
Recursive models have been introduced for describing the different stages of such sublayer formation (sometimes also referred to as the multiple X-point reconnection stage) and how many plasmoids are generated during the fully nonlinear evolution (Daughton et al. 2009; Huang \& Bhattacharjee 2010; Uzdensky et al. 2010; Ji \& Daughton 2011). To this end, it is convenient now to label with an $n$ the (half) length $L_{n}$, thickness $a_{n}$, and the local Lundquist number $S_{n}=\left(L_{n} / L\right) S$ of the $n$th current sheet. Previous models assume that at each step current sheets become unstable giving rise to smaller ones with length $L_{n}=L_{n-1} / N_{n-1}$, where $N_{n}$ is the number of plasmoids at step $n$ and thickness defined by the SP scaling, therefore $a_{n}=L_{n} S_{n}^{-1 / 2}$. In particular, Ji \& Daughton (2011) used a phenomenological scaling for the number of plasmoids $N_{n}=\left(S_{n} / S_{c}\right)^{\beta}$, where $\beta$ is left as a free parameter, and $S_{c} \simeq 10^{4}$ is the empirical critical Lundquist number for onset of plasmoid instability; such a scaling describing the number of plasmoids has been commonly adopted by other authors as well, motivated by the linear theory - although, strictly speaking, the linear theory as developed does not predict a renormalization to $S_{c}$, and therefore it yields a far larger value for $N_{n}$. Those assumptions lead to an infinite hierarchy, where secondary current sheets never cross the presumed stability threshold $S_{n} \lesssim S_{c}$, and for this reason Ji \& Daughton (2011) impose a minimum number of plasmoids $N_{\min }$ as a cutoff to find the maximum index of the hierarchy, $n_{*}$. Assuming also that each current sheet becomes unstable, they find that the total, final number $\mathcal{N}$ of plasmoids is $\mathcal{N} \simeq\left(S / S_{c}\right)^{z}$, with $z \sim 0.76,0.96$ for $n_{*} \simeq 3,2$ and $\beta=3 / 8,0.8$. This heuristic argument then leads to values for the number of plasmoids consistent with that found directly in numerical simulations (Cassak, Shay \& Drake 2009; Daughton et al. 2009; Huang \& Bhattacharjee 2010).

Before illustrating our recursive model, it is worth commenting on the question of the scaling of the number of islands with the Lundquist number as the plasmoid instability develops. Numerical simulations appear to have shown a number compatible with the scaling of the plasmoid instability on Sweet-Parker sheets, although generally speaking simulations tend to have Lundquist numbers which, already at the macroscopic scale, are quite close to the critical one for stability threshold $\left(S_{c}\right)$ (Bhattacharjee et al. 2009; Huang \& Bhattacharjee 2010). In this sense it is surprising that a scaling relationship such as $N_{S P} \sim S^{3 / 8}$, derived under the assumptions that flows may be completely neglected, might hold true. The same might be said of the scaling of islands along the sheet once nonlinearities become important, where results closer to $\mathcal{N} \sim S$ are found (Huang \& Bhattacharjee 2010). The reason is that counting $\mathrm{X}$-points is equivalent to precisely determining the topology of the magnetic field at very small scales, while the (maximum possible) value of the Lundquist number $S$ is essentially determined by the number of grid points. A scaling $\mathcal{N} \sim S$ along an extended one-dimensional sheet implies that the number of islands grows linearly with resolution. In such a reconnection configuration, the line is the original central current sheet neutral line: when moving to more general two-dimensional configurations, very high resolution simulations of fully developed turbulence show a number of $\mathrm{X}$-points appearing to scale as $\mathcal{N} \sim S^{3 / 2}$ (Wan et al. 2013). Following our previous statement, the corresponding upper limit set by resolution on the scaling of the number of island would be $\mathcal{N} \sim S^{2}$, so it appears that the scaling found by Wan et al. (2013) is reasonable. In other words, as stated by Wan et al. (2013) '[...] lack of adequate numerical resolution can easily increase the number of detected $\mathrm{X}$-points, thus producing non-physical results. Generally speaking, one requires high spatial resolution, to at least three times the Kolmogorov dissipation wavenumber, when using the pseudo-spectral approach that we have employed.' 
Here we reconsider the recursive reconnection model, but following more in detail the 'ideal' tearing scenario guided by our numerical results. We point out that a hierarchy of sublayers form anywhere in regions where currents have room to collapse. In this regard, boundary conditions may play a fundamental role, if the system size is not large enough to allow the dynamics to develop freely. Nevertheless, this limitation is automatically overcome in simulations of ideally unstable current sheets, since the wavelength of the ideal mode, $\lambda_{i} \sim 2 \pi S^{-1 / 6}$, is much smaller than the length of the current sheet (approximately given by the size of the simulation domain). Of course, the collapse of X-points cannot and does not occur uniformly along the sheet, rather, nonlinear evolution is determined by the competition and interplay between multiple recursive $\mathrm{X}$-point formation within the most intense currents, and an inverse cascade of merging plasmoids, as can also be seen by inspection of figure 7. Therefore, differently from the models discussed above, we apply the recursive idea to a given secondary current sheet, so that our model leaves the final number of plasmoids generated undefined. It instead provides a prediction of the total number of steps $n_{*}$ and of the time scale of the recursive process itself.

Since tearing is a multi-scale process, in the sense that it involves the formation of an inner singular layer, we also introduce the thickness of such inner layer $\delta_{n}$ of the $n$th unstable current sheet (for us $n \geqslant 1$ ), and we define the local Alfvén time $\tau_{A, n}=\left(L_{n} / L\right) \tau_{A}$. We rely on the following assumptions based on observations of our simulation results: first, that current sheets are lengthening and that they become unstable when the local critical aspect ratio is reached; second, we do not make any assumption on the length $L_{n}$, as is done in other recursive models (Shibata \& Tanuma 2001; Ji \& Daughton 2011), but, on the contrary, we observe that the thickness $a_{n}$ corresponds to the inner diffusion layer of the $(n-1)$ th tearing, $\delta_{n-1}$. These two requirements translate respectively into

$$
\frac{a_{n}}{L_{n}} \sim S_{n}^{-1 / 3}, \quad \frac{a_{n}}{L_{n-1}} \sim S_{n-1}^{-1 / 2},
$$

where for simplicity we have neglected the stabilizing effect of viscosity and of plasma flows, but which should be retained for the lower values of $S_{n}$ (see $\S 3.1$ ). Expressions given in (5.1) yield the following scaling laws:

$$
L_{n}=L S^{-1+(3 / 4)^{n}}, \quad \tau_{A, n}=\frac{L_{n}}{L} \tau_{A}, \quad S_{n}=S^{(3 / 4)^{n}} .
$$

Considering typical coronal conditions, for which $L \simeq 10^{9} \mathrm{~cm}, B_{0} \simeq 50 \mathrm{G}$, the number density $n_{0} \simeq 10^{9} \mathrm{~cm}^{-3}$ and the temperature $T \simeq 10^{6} \mathrm{~K}$, then the macroscopic Lundquist number is $S \simeq 10^{13}$ (Braginkii 1965). The sequences of $L_{n}$ and of $S_{n}$ for $S=10^{13}$ are represented in figure 12 , showing that the formation of microscopic scales occurs very rapidly. In this case, after a number of steps $n_{*} \simeq 4$ the local Lundquist number reaches $S_{n_{*}} \simeq 10^{4}$, the region close to where one expects to find complete flow-driven stabilization. The time required to reach this marginally stable state gives an indication of the time scale for the complete disruption of the original current sheet, and is given by the time required to trigger the first instability $\tau_{0}\left(\tau_{0} \simeq \tau_{i}\right)$ plus the time of the recursive reconnection, that for $S=10^{13}$ is about

$$
\tau \simeq \tau_{0}+\sum_{n=1}^{n_{*}} \tau_{A, n} \simeq(10-100) \tau_{A}+5 \times 10^{-4} \tau_{A} .
$$


(a)

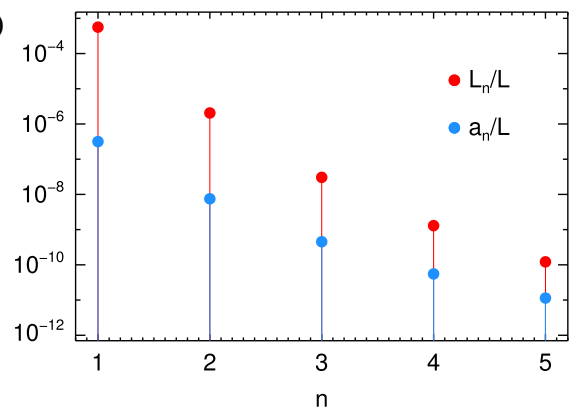

(b)

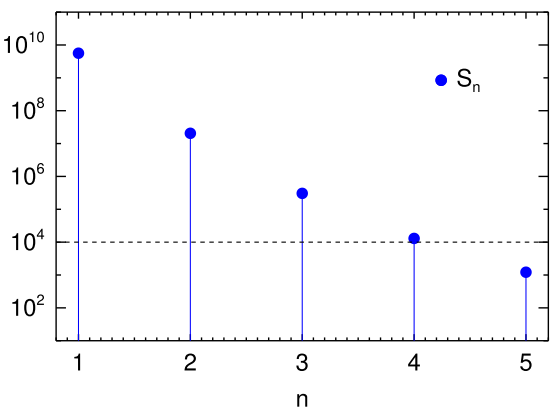

FIgURE 12. Plot of the sequence $L_{n} / L(a)$ and $S_{n}(b)$ for $S=10^{13}$, see (5.2).

Formation of smaller scales can mediate the transition to a Hall (or kinetic) reconnection regime, when the ion scales are reached dynamically (Cassak et al. 2005; Daughton et al. 2009; Shepherd \& Cassak 2010; Huang et al. 2011). If ion scales are formed during the recursive reconnection, e.g. $a_{n} \simeq d_{i}$, then different scalings should be adopted from that moment on. For instance, with the parameters chosen above as representative of the solar corona, the normalized ion inertial length turns out to be about $d_{i} / L \simeq 10^{-7}$, which is of the same order of the thickness formed at the first step, $a_{1} / L$, for $S=10^{13}$. A study including the Hall effect is however necessary to assess with more precision at which thickness the MHD scalings used so far are modified. The main point here is that regardless of whether MHD or a specific kinetic regime is the more suitable model, 'ideal' tearing provides a clear well-defined threshold for the onset of fast reconnection (Del Sarto et al. 2016), so that (5.2) can be extended to kinetic regimes as well.

\section{Comparison with previous models}

In spite of the different initial conditions, the evolution of the current sheet formed during the nonlinear stage of the 'ideal' tearing, as shown for instance in figure 9, is qualitatively reminiscent of, and can be compared to the 'embedded reconnection' scenario (Shay et al. 2004; Cassak \& Drake 2009), in which the diffusion layer is embedded within a thicker underlying current sheet. The argument of the embedded layer led Cassak \& Drake (2009) to take into account the increasing Alfvén speed due to pile up just upstream the inner diffusion layer, and to find in this way the scaling $a_{1} / L_{1} \sim S_{1}^{-1 / 3}$ for onset of instability (in order to avoid confusion, we adopt here our notation, and $\left.S_{1}=v_{A} L_{1} / \eta\right)$. Nevertheless, their scaling has a different origin and it stems from the criterion to destabilize a SP sheet, when the local Lundquist number exceeds the value $S_{c}=10^{4}$. Cassak \& Drake (2009) assumed that the diffusion layer ultimately becomes an unstable SP sheet, which however satisfies $a_{1} / L_{1} \sim S_{u p}^{-1 / 2}, S_{u p}$ being the upstream Lundquist number, defined through the upstream magnetic field $B_{u p}$ and $L_{1} ; B_{u p}$ can be approximated near the neutral line by linearizing the magnetic field, hence $B_{u p} \simeq B_{0}\left(a_{1} / a\right)$. Direct substitution of $B_{u p}$ into the SP scaling for the inverse aspect ratio yields the same exponent $\alpha=1 / 3$ for onset of instability. On the other hand, the 'ideal' tearing scaling derives from requiring an $S$-independent growth rate from the complete eigenmode analysis of tearing instability, and the presence of flows modifies the scaling exponent $\alpha$ according to (3.3). For their values $S_{1} \simeq 2.5 \times 10^{4}-10^{5}$ the exponent $\alpha_{c} \simeq 0.48-0.46$, that give an unstable current sheet when $a_{1} / L_{1} \sim S_{1}^{-\alpha_{c}} \simeq 0.007-0.005$. These estimates are in agreement with the unstable inverse aspect ratios found in the Cassak \& Drake (2009) paper. 
It is interesting to note that the scaling exponent $\alpha=1 / 3$ as an instability criterion was also derived by Shibata \& Tanuma (2001) in the framework of 'fractal' reconnection, introduced for the first time by those authors as a possible phenomenological model for current sheet disruption during solar flares. Based on the argument of Biskamp (1986), Shibata \& Tanuma (2001) searched for a condition to overcome the stabilizing effect of flows on the tearing instability. They concluded that, given a current sheet of inverse aspect ratio $a_{n} / L_{n}$, the maximum growth rate of the tearing had to exceed the plasmoid evacuation rate $\Gamma_{n} \simeq v_{A} / L_{n}=1 / \tau_{A, n}$ for the tearing to develop (in the end, effects of flows on the growth rate are not taken into account in their model). Shibata \& Tanuma (2001) can find in this way that the current sheet must at least have an inverse aspect ratio $a_{n} / L_{n} \leqslant S_{n}^{-1 / 3}$ in order to become unstable. Again, the result of Shibata \& Tanuma (2001) stems from a different line of thought, as they searched for a maximum growth rate larger than the inverse of the ideal time $\tau_{A, n}$, and not for an 'ideal' growth rate, independent from the Lundquist number. Next, in their 'fractal' model the authors assume that the length for each sublayer corresponds to the wavelength of the fastest growing mode, i.e. $L_{n}=2 \pi / k_{n}$, that yields a different scaling law for $L_{n}$ with respect to ours, having a weaker dependence on $n$.

\section{Summary and discussion}

We have given an overview of linear tearing instabilities within thin current sheets at arbitrary aspect ratios in resistive MHD and discussed some extensions, namely, Reduced MHD with inclusion of kinetic effects. Theory shows that fast reconnection, developing on time scales independent of the non-ideal terms in Ohm's law, sets in at a critical aspect ratio $L / a_{i}$ that satisfies specific scaling laws with plasma parameters such as $S$ and $d_{e} / L$. Such scaling laws can be modified by viscosity at large Prandtl numbers, or by kinetic effects when the inner diffusion layer is of the order of, or smaller than, $\rho_{s}$ and $\rho_{i}$ (Pucci \& Velli 2014; Tenerani et al. 2015a; Del Sarto et al. 2016). The main result that emerges from these studies is that in the asymptotic limit $S \rightarrow \infty$ or $d_{e} / L \rightarrow 0$ a violent transition from a stable state (reconnecting over infinitely long times) to an ideally unstable one has to occur when approaching the critical thickness $a_{i} / L$ from above. The onset of fast reconnection cannot take place within current sheets that are thicker than critical, for sufficiently large values of $S$ : unstable modes at smaller aspect ratios, $L / a<L / a_{i}$, have growth rates whose values tend to zero in the asymptotic limit, whereas, precisely at $L / a_{i}$, the full dispersion relation $\gamma(k)$ becomes rapidly peaked around $k_{m}$ (cf. for instance, figure $5 b$ ), the 'ideal' mode being the only surviving one in the asymptotic limit; 'ideal' tearing onset, by breaking up the current on an ideal time scale, prevents the spontaneous formation of much larger aspect ratio current sheets, such as the Sweet-Parker one. Fully nonlinear MHD simulations show that the inner diffusion layer of the ideally reconnecting current sheet evolves during the nonlinear stage into an elongated secondary current sheet, that in turn becomes unstable to 'ideal' tearing at the local Alfvén time. This process of current sheet formation from of the inner diffusion layer proceeds in a recursive way at smaller and smaller scales, that can be accounted for by properly rescaling 'ideal' tearing to the local length of the sheet (Landi et al. 2015; Tenerani et al. 2015b), even though an appropriate statistical study of the fully nonlinear stage has not been completed yet because of the incredibly high resolutions required to resolve the recursive formation of plasmoids down to the dissipative scales. 
In the solar corona, where inter-species collisions usually provide the dominant dissipation mechanism for reconnection, the Lundquist numbers are $S \simeq 10^{12}-10^{14}$. For instance, for $S=10^{13}$ the critical inverse aspect ratio is $a_{i} / L \sim S^{-1 / 3} \simeq 5 \times 10^{-5}$, i.e. for a loop structure of length $L \simeq 10^{9} \mathrm{~cm}$ the critical thickness would be $a_{i} \simeq 500 \mathrm{~m}$, with an inner layer of about $\delta_{i} \simeq 3 \mathrm{~m}$, intermediate between the ion inertial length, $d_{i} \simeq 10 \mathrm{~m}$, and the ion Larmor radius, $\rho_{i} \simeq 10 \mathrm{~cm}$. Two-fluid effects related to the Hall term in the generalized Ohm's law need therefore to be investigated to give a more realistic description of ideally unstable current sheets. Inclusion of such effects becomes however necessary when nonlinearities naturally lead to the formation of microscopic scales, e.g. during recursive reconnection.

A different regime describes the Earth's magnetosphere, and in particular the magnetotail, for which a kinetic description is more suitable (Coppi, Laval \& Pellat 1966; Schindler 1974; Vasyliunas 1975; Daughton 1999; Sitnov et al. 2014). Here, resistive reconnection, due either to inter-specie collisions or to wave-particle scattering (Coroniti 1985), is usually dominated by reconnection induced by electron inertia. Assuming values of plasma density $n_{0} \simeq 0.1 \mathrm{~cm}^{-3}$, electron temperature $T_{e} \simeq 10^{7} \mathrm{~K}$ (for simplicity we consider ions and electrons at the same temperature) and magnetic field $B_{0} \simeq 10^{-4} \mathrm{G}$, and taking as typical sheet length $L \simeq 10^{9} \mathrm{~cm}$, then the Lundquist number ranges from $S \simeq 10^{10}$ (for wave-particle scattering; Eastwood et al. (2009)) to $S \simeq 10^{15}$ (for standard collisions), whereas $d_{e}^{2} / L^{2} \simeq 10^{-6}$. The electron skin depth is about $d_{e} \simeq 10 \mathrm{~km}$ and the ion length scales are $\rho_{i} \simeq \rho_{s} \simeq 300 \mathrm{~km}$, of the order of the ion skin depth, $d_{i} \simeq 700 \mathrm{~km}$. Ionic scales are not far from the thickness of the central current sheet, that is observed to thin down to about $1000 \mathrm{~km}$ during the substorm growth phase, and for these parameters the kinetic equation (3.9) predicts $a_{i} \simeq 400 \mathrm{~km}$. However, what is the effective driving mechanism for collisionless reconnection, and which are the dominant kinetic effects to be retained, are still matter of debate and the models that we have discussed can provide only indicative estimates of the critical thickness there (recall also that they require a strong guide field, which is rarely observed in magnetotail). The collisionless model that we have considered includes some kinetic effects, namely, FLR corrections to a dominant gyrotropic dynamics, but it does not include Landau resonances and the electron pressure anisotropy, which instead are known to allow reconnection (Coppi et al. 1966; Schindler 1974; Vasyliunas 1975; Cai \& Lee 1997). Other kinetic effects relevant for the magnetotail configuration should be considered as well, including electron meandering orbits around the neutral line in the absence of strong a guide field (Sonnerup 1971), or particle temperature anisotropies which are known to affect reconnection rates (Chen \& Palmadesso 1983; Karimabadi, Daughton \& Quest 2004; Matteini et al. 2013). In this regard, it is still unclear in which way more realistic equilibria having a magnetic field component parallel to the shear, that is, two-dimensional equilibria rather than a simple Harris sheet, impact the stability of the current sheet itself, and in particular the possible onset of kinetic tearing modes (Sitnov et al. 2014; Pritchett 2015). These problematics need to be further explored.

In the present discussion we have not considered three-dimensional effects, which allow new instabilities and may affect both the linear (Daughton 1999; Baarlud, Battacharjee \& Huang 2012) and especially the nonlinear evolution (Dahlburg \& Einaudi 2002; Landi et al. 2008; Daughton et al. 2011) of tearing modes, allowing a much richer dynamical evolution than in two dimensions. Magnetic islands extend in the third direction giving rise to flux ropes, that in turn are subject to secondary ideal instabilities. If the guide field is not sufficiently strong (Dahlburg, Klimchuk \& Antiochos 2005), flux ropes start to braid among themselves, by overcoming in the 
typical two-dimensional evolution characterized by magnetic island coalescence and $\mathrm{X}$-point collapse. By favouring in this way secondary reconnection within multiple layers along the ropes, the nonlinear dynamics efficiently drives the system into a turbulent state. It is therefore of interest to study how our onset scenario, but especially the nonlinear recursive reconnection model, change in three-dimensions.

\section{Acknowledgements}

This work was supported by the NASA program LWS, grant NNX13AF81G. M.V. acknowledges support via the NASA Solar Probe Plus observatory scientist grant. The authors thank the editors and R. Lionello and F. Pegoraro for their useful comments and suggestions. Computational resources supporting this work were provided by the NASA High-End Computing (HEC) Program through the NASA Advanced Supercomputing (NAS) Division at Ames Research Center.

\section{REFERENCES}

Adler, E. A., Kulsrud, R. M. \& White, R. B. 1980 Magnetic driving energy of the collisional tearing modes. Phys. Fluids 23, 1375-1379.

Ajello, M., Albert, A., Allafort, A., Baldini, L., Barbiellini, G., Bastieri, D., Bellazzini, R., Bissaldi, E., Bonamente, E., BRAndt, T. J. et al. 2014 Impulsive ad long duration high-energy gamma-ray emission from the very bright 2012 March 7 solar flares. Astrophys. J. 789, 20.

Akasofu, S.-I. 1964 The development of the auroral substorm. Planet. Space Sci. 12, 273-282.

Ali, A., JiQuan, Li \& Kishimoto, Y. 2014 On the abrupt growth dynamics of nonlinear resistive tearing mode and the viscosity effects. Phys. Plasmas 21, 052312.

Angelopoulos, V., Runov, A., Zhou, X.-Z., Turner, D. L., Kiehas, S. A., Li, S.-S. \& ShinohaRA, I. 2013 Electromagnetic energy conversion at reconnection fronts. Science 341, $1478-1482$.

ARA, G., BASU, B. \& COPPI, B. 1978 Magnetic reconnection and the $m=1$ oscillations in current carrying plasmas. Ann. Phys. 112, 443-476.

Aulanier, G., Pariat, E. \& Démoulin, P. 2005 Current sheet formation in quasi-separatrix layers and hyperbolic flux tubes. Astron. Astrophys. 444, 961-976.

BaARlud, S. D., BattacharJee, A. \& Huang, Y.-M. 2012 Reduced magnetohydrodynamic theory of oblique plasmoid instabilities. Phys. Plasmas 19, 022101.

Battacharjee, A., Germaschewski, K. \& NG, C. S. 2002 Current singularities: drivers of impulsive reconnection. Phys. Plasmas 12, 042305.

Battacharjee, A., Huang, Y.-M., Yang, H. \& Rogers, B. 2009 Fast reconnection in high -Lundquist-number plasmas due to the plasmoid instability. Phys. Plasmas 16, 112102,1-5.

Birn, J., Drake, J. F., Shay, M. A., Rogers, B. N., Denton, R. E. et al. 2001 Geospace Envirnmental Modeling (GEM) magnetic reconnection challenge. J. Geophys. Res. 106, 3715-3719.

Birn, J. \& SCHInder, K. 2002 Thin current sheets in the magnetotail and the loss of equilibrium. J. Geophys. Res. 107, 1117.

Biskamp, D. 1986 Magnetic reconnection via current sheets. Phys. Fluids 29, 1520-1531.

BISKAMP, D. 1993 Current sheet profiles in two dimensional magnetohydrodynamics. Phys. Plasmas 5, 3893-3896.

BISKAMP, D. 2000 Nonlinear Magnetohydrodynamics. Cambridge University Press.

BISKAMP, D., SCHWARTZ, E. \& DRAKE, J. F. 1995 Ion-controlled collisionless magnetic reconnection. Phys. Rev. Lett. 75, 3850-3853.

Bondeson, M. \& Sobel, J. R. 1984 Energy balance of the collisional tearing mode. Phys. Fluids 27, 2028.

BRAginskiI, S. I. 1965 Transport processes in a plasma. Rev. Plasma Phys. 1, 205. 
Bulanov, S. V., SyrovatskiǏ, S. I. \& SAKai, J. 1978 Stabilizing influence of plasma flow on dissipative tearing instability. J. Expl Theor. Phys. 28, 177.

Bulanov, S. V., SAKaI, J. \& SyrovatskiǏ, S. I. 1979 Tearing mode instability in approximately steady MHD configurations. Sov. J. Plasma Phys. 5, 157-173.

CAI, H.-J. \& LEE, L. C. 1997 The generalized Ohm's law in collisionless magnetic reconnection. Phys. Plasmas 4, 509.

CAssak, P. A. \& DRAKe, J. F. 2009 The impact of microscopic magnetic reconnection on pre-flare energy storage. Astrophys. J. Lett. 707, L128-L162.

CAssak, P. A. \& Drake, J. F. 2013 On the phase diagrams of magnetic reconnection. Phys. Plasmas 20, 061207,1-8.

Cassak, P. A., Shay, M. A. \& Drake, J. F. 2005 Catastrophe model for fast magnetic reconnection onset. Phys. Rev. Lett. 95, 235002.

Cassak, P. A., Shay, M. A. \& Drake, J. F. 2009 Scaling of Sweet-Parker reconnection with secondary islands. Phys. Plasmas 16, 120702.

Chen, J. \& Palmadesso, P. 1983 Tearing instability in an anisotropic neutral sheet (no. NRL-MR5178). Naval Research Lab Washington DC.

Comisso, L., Grasso, D., Waelbroeck, F. L. \& Borgogno, D. 2013 Gyro-induced acceleration of magnetic reconnection reconnection. Phys. Plasmas 20, 092118.

Comisso, L., Grasso, D. \& Waelbroeck, F. L. 2015 Extended theory of the Taylor problem in the plasmoid-unstable regime. Phys. Plasmas 22, 042109.

Coppi, B., Laval, G. \& Pellat, R. 1966 Dynamics of the geomagnetic tail. Phys. Rev. Lett. 16, 1207-1210.

Coppi, B., Galvao, R., Pellat, R., Rosenbluth, M. N. \& Rutherford, P. H. 1976 Resistive internal kink modes. Sov. J. Plasma Phys. 2, 533-535.

Coroniti, F. V. 1985 Space plasma turbulent dissipation: reality or myth? Space Sci. Rev. 42, 399-410.

DAhlburg, R. B. \& Einaudi, G. 2002 MHD unstable modes in the 3D evolution of 2D MHD structures and the dimished role of coalescence instabilities. Phys. Lett. A 294, 101-107.

Dahlburg, R. B., Klimchuk, J. A.\& Antiochos, S. K. 2005 An explanation for the 'switch-on' nature of magnetic energy release and its application to coronal heating. Astrophys. J. 622, 1191-1201.

Daughton, W. 1999 The unstable eigenmodes of a neutral sheet. Phys. Plasmas 6, 1329-1343.

DAughton, W. \& RoYtershteYN, V. 2012 Emerging parameter space map of magnetic reconnection in collisional and kinetic regimes. Space Sci. Rev. 172, 271-282.

Daughton, W., Roytershteyn, V., Albright, B. J., Karimabadi, H., Yin, L. \& Bowers, K. J. 2009 Transition from collisional to kinetic regimes in large-scale reconnection layers. Phys. Rev. Lett. 103, 065004.

Daughton, W., Roytershteyn, V., Karimabadi, H., Yin, L., Albright, B. J., Bergen, B. \& BOWERS, K. J. 2011 Role of electron physics in the development of turbulent magnetic reconnection in collisionless plasmas. Nat. Phys. 7, 539-542.

Daughton, W., Scudder, J. \& Karimabadi, H. 2006 Fully kinetic simulations of undriven magnetic reconnection with open boundary conditions. Phys. Plasmas 13, 072101.

Del Sarto, D., Califano, F. \& Pegoraro, F. 2006 Electron parallel compressibility in the nonlinear development of two-dimensional collisionless magnetohydrodynamic reconnection. Mod. Phys. Lett. B 20, 931-961.

Del Sarto, D., Pucci, F., Tenerani, A. \& Velli, M. 2016 'Ideal' tearing and the transition to fast reconnection in the weakly collisional MHD and EMHD regimes. J. Geophys. Res. 121, $1857-1873$.

Dobrott, D., Prager, S. C. \& TAYlor, J. B. 1977 Influence of diffusion on the resistive tearing mode. Phys. Fluids 20, 1850-1854.

Drake, J. F., Shay, M. A. \& Swisdak, M. 2008 The Hall fields and fast magnetic reconnection. Phys. Plasmas 15, 042306.

Eastwood, J. P., Phan, T. D., Bale, S. D. \& Tuulin, A. 2009 Observation of turbulence generated by magnetic reconnection. Phys. Rev. Lett. 102, 035001. 
Ellison, M. A. 1946 Visual and spectrographic observations of a great solar flare, 1946 July 25. Mon. Not. R. Astron. Soc. 106, 500.

Fletcher, L., Dennis, B. R., Hudson, H. S., KrucKer, S. et al. 2011 An observational overview of solar flares. Space Sci. Rev. 20, 19-106.

Furth, H. P., Killeen, J. \& Rosenbluth, M. N. 1963 Finite resistivity instabilities of a sheet pinch. Phys. Fluids 20, 459-484.

Grasso, D., Hastie, R. J., Porcelli, F. \& Tebaldi, C. 2008 Critical for stability of visco-resistive tearing modes. Phys. Plasmas 15, 072113.

Grasso, D., TAssi, E. \& WAelbroeck, F. L. 2010 Nonlinear gyrofluid simulations of collisionless reconnection. Phys. Plasmas 17, 082312.

Hsieh, M.-S. \& OTto, A. 2015 Thin current sheet formation in response to the loading and the depletion of magnetic flux during the substorm growth phase. J. Geophys. Res. 120, 4264-4278.

HuAng, Y.-M. \& Bhattacharjee, A. 2010 Scaling laws of resistive magnetohydrodynamic reconnection in the high-Lundquist-number, plasmoid-unstable regime. Phys. Plasmas 17, 062104.

Huang, Y.-M. \& BhattacharJee, A. 2013 Plasmoid instability in high-Lundquist-number magnetic reconnection. Phys. Plasmas 20, 055702.

Huang, Y.-M., Bhattacharjee, A. \& Sullivan, B. P. 2011 Onset of fast reconnection in Hall magnetohydrodynamics mediated by the plasmoid instability. Phys. Plasmas 18, 072109.

Jemella, B. D., Shay, M. A., Drake, J. F. \& Rogers, B. N. 2003 Impact of frustrated singularities on magnetic island evolution. Phys. Rev. Lett. 91, 125002.

Ji, H. \& DAghton, W. 2011 IPhase diagram for magnetic reconnection in heliophysical, astrophysical, and laboratory plasma. Phys. Plasmas 18, 111207.

Kadomtsev, B. B. 1975 Disruptive instability in Tokamaks. Sov. J. Plasma Phys. 1, 389-391.

KANE, S. R. 1974 Impulsive (flash) phase of solar flares: hard X-ray, microwaves, EUV and optical observations. In Coronal Disturbances; Proceedings of the Symposium, Surfer's Paradise, Queensland, Australia, September 7-11, 1973 (A75-37351 17-92), pp. 105-141. Dordrecht, D. Reidel Publishing Co..

Karimabadi, H., Daughton, W. \& Quest, K. B. 2004 Role of electron temperature anisotropy in the onset of magnetic reconnection. Geophys. Res. Lett. 31, L18801.

Klimas, A., Hesse, M. \& Zenitani, S. 2008 Particle-in-cell simulation of collisionless reconnection with open outflow boundaries. Phys. Plasmas 15, 082102.

Landi, S., Del Zanna, L., PAPIni, E., PuCCI, F. \& Velli, M. 2015 Resistive Magnetohydrodynamics simulations of the ideal tearing mode. Astrophys. J. 806, 131,1-8.

Landi, S., Londrillo, P., Velli, M. \& Bettarini, L. 2008 Three-dimensional simulations of compressible tearing instability. Phys. Plasmas 15, 012302.

LAPENTA, G. 2008 Self-feeding turbulent magnetic reconnection on macroscopic scale. Phys. Rev. Lett. 100, 23500.

Lentini, M. \& Pereyra, V. 1974 A variable order finite difference method for nonlinear multipoint boundary value problems. Maths Comput. 28, 9811004.

LiU, Y.-H., Daughton, W., Karimabadi, H., Li, H. \& Peter, G. S. 2014 Do disperisve waves play a role in collisionless magnetic reconnection? Phys. Plasmas 21, 022113.

Loureiro, N. F., Cowley, S. C., Dorland, W. D., Haines, M. G. \& Schekochihin, A. A. 2005 X-Point collapse and saturation in the nonlinear tearing mode reconnection. Phys. Rev. Lett. 95, 235003.

Loureiro, N. F., Samtaney, R., Schekochinin, A. A. \& Uzdensky, D. A. 2012 Magnetic reconnection and stochastic plasmoid chains in high-Lundquist-number plasmas. Phys. Plasmas 19, $042303,1-5$.

Loureiro, N. F., Schekochinin, A. A. \& Uzdensky, D. A. 2007 Instability of current sheets and formation of plasmoid chains. Phys. Plasmas 14, 100703,1-4.

Loureiro, N. F., Schekochihin, A. A. \& Uzdensky, D. A. 2013 Plasmoid and Kelvin-Helmholtz instabilities in Sweet-Parker current sheets. Phys. Rev. E 87, 013102. 
Loureiro, N. F. \& Uzdensky, D. A. 2015 From the Sweet-Parker model to stochastic plasmoid chains. Plasma Phys. Control. Fusion 58, 014021.

Malara, F., Veltri, P. \& Carbone, V. 1992 Competition among nonlinear effects in tearing instability saturation. Phys. Fluids B 4, 3072-3086.

Mandt, M. E., Denton, R. E. \& Drake, J. F. 1994 Transition to whistler mediated reconnection. Geophys. Res. Lett. 21, 73-76.

Masuda, S., Kosugi, T., Hara, H., Tsuneta, S. \& Ogawara, Y. 1994 A loop-top hard X-ray source in a compact solar flare as evidence for magnetic reconnection. Nature 371, 495-497.

Matteini, L., Landi, S., Velli, M. \& Matthaeus, W. H. 2013 Proton temperature anisotropy and magnetic reconnection in the solar wind: effects of kinetic instabilities on current sheet stability. Astrophys. J. 763, 142.

Matthaeus, W. H. \& Lamkin, S. L. 1985 Rapid magnetic reconnection caused by finite amplitude fluctuations. Phys. Fluids 28, 303.

Militello, F., Borgogno, D., Grasso, D., Marchetto, C.\& Ottaviani, M. 2011 Asymmetric tearing mode in the presence of viscosity. Phys. Plasmas 18, 112108.

NI, L., KLIEM, B., LiN, J. \& WU, N. Fast magnetic reconenction in the solar chromosphere mediated by the plasmoid instability. Astrophys. J. 799, 79.

Ottaviani, M. \& Porcelli, F. 1993 Fast nonlinear magnetic reconnection. Phys. Plasmas. 2, 4104.

Park, W., Monticello, D. A. \& White, R. B. 1984 Reconnection rates of magnetic fields including the effects of viscosity. Phys. Fluids 27, 147-149.

PARKER, E. N. 1957 Sweet's mechanism for merging magnetic fields in conducting fluids. J. Geophys. Res. 62, 509-520.

PARKER, E. N. 1988 Nanoflares and the solar X-ray corona. Astrophys. J. 330, 474-479.

Pegoraro, F. \& SCheP, T. J. 1986 Theory of resistive modes in the balloning representation. Plasma Phys. Contol. Fusion 28, 647.

PetscheK, H. E. 1964 Magnetic field annihilation. In The Physics of Solar Flares, Proceedings of the AAS-NASA Symposium held 28-30 October, 1963 at the Goddard Space Flight Center, Greenbelt, $M D$ (ed. W. N. Hess), p. 425. National Aeronautics and Space Administration, Science and Technical Information Division.

Porcelli, F. 1987 Viscous resistive magnetic reconnection. Phys. Fluids 30, 1734-1742.

Porcelli, F. 1991 Collisionless $m=1$ tearing mode. Phys. Rev. Lett. 66, 425.

Porcelli, F., Borgogno, D., Califano, F., Grasso, D., Ottaviani, M. \& Pegoraro, F. 2002 Recent advances in collisionless magnetic reconnection. Plasma Phys. Control. Fusion 44, B389-B405.

PRITCHETT, P. L. 2015 Instability of current sheets with a localized accumulation of magnetic flux. Phys. Plasmas 22, 062102.

PucCI, F. \& Velli, M. 2014 Reconnection of quasi-singular current sheets: the 'ideal' tearing mode. Astrophys. J. Lett. 780, L19,1-4.

Rappazzo, A. F. \& PARKer, E. N. 2013 Current sheets formation in tangled coronal magnetic fields. Astrophys. J. Lett. 773, L2.

Rappazzo, A. F., Velli, M., Einaudi, G. \& Dahlburg, R. B. 2008 Nonlinear dynamics of the Parker scenario for coronal heating. Astrophys. J. 677, 1348.

Rogers, B. N., Denton, R E., Drake, J. F. \& Shay, M. A. 2001 Role of dispersive waves in collisionless magnetic reconnection. Phys. Rev. Lett. 87, 195004.

Rutherford, P. H. 1973 Nonlinear growth of the tearing mode. Phys. Fluids 16, 1903.

Samtaney, R., Loureiro, N. F., UzDensky, D. A., Schekochinin, A. A. et al. 2009 Formation of plasmoid chains in magnetic reconnection. Phys. Rev. Lett. 103, 105004.

Sanny, J., McPherron, R. L., Russel, C. T., Baker, D. N., Pulkkinen, T. I. \& Nishida, A. 1994 Growth phase thinning of the near-Earth current sheet during the CDAW 6 substorm. $J$. Geophys. Res. 99, 5905-5816.

Schekochinin, A. A., Cowley, S. C., Kulsrud, R. M., Hammett, G. W. \& Sharma, P. 2005 Plasma instabilities and magnetic field growth in cluster of galaxies. Astrophys. J. 629, 139.

Schep, T. J., Pegoraro, F. \& Kuvshinov, B. N. 1994 Generalized two-fluid theory of nonlinear magnetic structures. Phys. Plasmas. 1, 2843. 
SCHINDler, K. 1974 A theory of the substorm mechanism. J. Geophys. Res. 79, 2803.

Scudder, J. D., Karimabadi, H., Daughton, W. \& Roytershteyn, V. 2014 Frozen flux violation, electron demagnetization and magnetic reconnection. Phys. Plasmas 22, 101204.

Shay, M. A., Drake, J. F., Rogers, B. N. \& Denton, R. E. 1999 The scaling of collisionless, magnetic reconnection for large systems. Geophys. Res. Lett. 26, 2163-2166.

Shay, M. A., Drake, J. F., Swisdak, M. \& Rogers, B. N. 2004 The scaling of embedded collisionless reconnection. Phys. Plasmas 11, 2199-2213.

SHEPHERD, L. S. \& CASSAK, P. A. 2010 Comparison of secondary islands in collisional reconnection to Hall reconnection. Phys. Rev. Lett. 105, 015004.

Shibata, K. \& TANuma, S. 2001 Plasmoid-induced-reconnection and fractal reconnection. Earth Planets Space 53, 473-482.

Sitnov, M., Merkin, V. G., Swisdak, M., Motoba, T., Buzulukova, N., Moore, T. E., MAUK, B. H. \& OHTANI, S. 2014 Magnetic reconnection, buoyancy, and flapping motions in magnetotail explosions. J. Geophys. Res. Space Phys. 119, 7151-7168.

SONNERUP, B. U. Ö. 1971 Adiabatic particle orbits in a magnetic null sheet. J. Geophys. Res. 76, $8211-8222$.

STRAUSS, H. R. 1976 Nonlinear, three-dimensional magnetohydrodynamics of noncircular tokamaks. Phys. Fluids 19, 134-140.

Su, Y., Veronig, A. M., Holman, G. D., Dennis, B. R., Wang, T., Temmer, M. \& Gan, W. 2013 Imaging coronal magnetic-field reconnection in a solar flare. Nat. Phys. 9, 489-493.

Sulem, P. L., Frisch, U., Pouquet, A.\& MeneguzzI, M 1985 On the exponential flattening of current sheets near neutral X-points in two-dimensional ideal MHD flow. J. Plasma Phys. 33, 191-198.

SweEt, P. A. 1958 The neutral point theory of solar flares. In Electromagnetic Phenomena in Cosmical Physics (IAU Symposium), vol. 6, pp. 123-134. Cambridge University Press.

SYROVATSKIII, S. I. 1971 Formation of current sheets in a plasma with frozen-in strong magnetic field. Sov. Phys. JETP 33, 933-940.

Tenerani, A., Rappazzo, A. F., Velli, M. \& Pucci, F. $2015 a$ The tearing mode instability of thin current sheets: the transition to fast reconnection in the presence of viscosity. Astrophys. J. 801, 145,1-7.

Tenerani, A., Velli, M., Rappazzo, A. F. \& Pucci, F. 2015b Magnetic reconnection: recursive current sheet collapse triggered by 'idel' tearing. Astrophys. J. Lett. 813, L32.

Terasawa, T. 1983 The Hall effect on tearing mode instability. Geophys. Res. Lett. 10, 475-478.

Titov, V. S., GalsgaARD, K. \& NeUKirch, T. 2003 Magnetic pinching of hyperbolic flux tubes. I. Basic Estimations. Astrophys. J. 582, 1172.

UzDEnsky, D. A. \& Loureiro, N. F. 2016 Magnetic reconnection onset via disruption of a forming current sheet by the tearing Instability. Phys. Rev. Lett. 116, 105003.

Uzdensky, D. A., Loureiro, N. F. \& SCheKochinin, A. A. 2010 Fast magnetic reconnection in the plasmoid-dominated regime. Phys. Rev. Lett. 105, 235002.

Vasyliunas, V. M. 1975 Theoretical models of magnetic field line merging. Rev. Geophys. 13, 303-336.

Venstein, G. \& Kusano, K. 2015 Nonlinear regimes of forced magnetic reconnection. Phys. Plasmas 22, 090707.

WAelbroeck, F. L. 1989 Current sheets and nonlinear growth of the $m=1$ kink-tearing mode. Phys. Plasmas 2, 2372-2380.

Waelbroeck, F. L., Hazeltine, R. D. \& Morrison, P. J. 2009 A Hamiltonian electromagnetic gyrofluid model. Phys. Plasmas 16, 032109.

Wan, M., Mettheaus, W. H., Servidio, S.\& Oughton, S. 2013 Generation of X-points and secondary islands in 2D magnetohydrodynamic turbulence. Phys. Plasmas 20, 042307.

Wesson, J. A. 1986 Sawtooth oscillations. Plasma Phys. Control. Fusion 28, 243-248.

Yamada, M., Kulsrud, R. \& Ji, H. 2010 Magnetic reconnection. Rev. Mod. Phys. 82, 603.

Yamada, M., Levinton, F. M., Pompherey, N., Budny, R., Manickam, J. \& Nagayama, Y. 1994 Investigation of magnetic reconnection during a sawtooth crash in a high-temperature tokamak plasma. Phys. Plasmas 1, 3269. 


\section{Dr. Anna Tenerani}

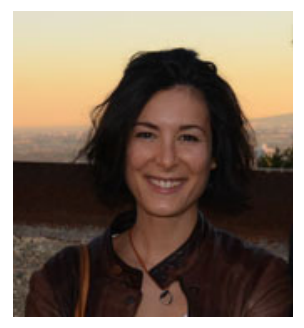

Dr. Anna Tenerani graduated in Physics at the University of Pisa in 2009 with Prof. F. Califano as advisor, before continuing on for her graduate studies in Astrophysics through the joint French-Italian Ph.D. program, between the University of Pierre et Marie Curie (Paris) and the University of Pisa. She obtained her Ph.D. degree in 2012, under the supervision of Dr. O. Le Contel and Prof. F. Califano, working on observations and numerical simulations of plasma waves and instabilities in the Earth's magnetosphere. She then held a Caltech post-doctoral scholarship, working with Prof. M. Velli at JPL, before continuing on as a research scientist at UCLA working on solar wind turbulence and magnetic reconnection 